Portland State University

PDXScholar

$5-1-1969$

\title{
A method of measuring the electron energy losses in transmission through thin films
}

John Patrick Stewart

Portland State University

Follow this and additional works at: https://pdxscholar.library.pdx.edu/open_access_etds

Let us know how access to this document benefits you.

\section{Recommended Citation}

Stewart, John Patrick, "A method of measuring the electron energy losses in transmission through thin films" (1969). Dissertations and Theses. Paper 908.

https://doi.org/10.15760/etd.908

This Thesis is brought to you for free and open access. It has been accepted for inclusion in Dissertations and Theses by an authorized administrator of PDXScholar. Please contact us if we can make this document more accessible: pdxscholar@pdx.edu. 
AN ABSTRACT OF THE THESIS OF John Patrick Stewart for the Master of Science degree in Physics presented on June 24, 1968.

Title: A Method of Measuring the Electron Energy Losses in Transmission Through Thin Films

APPROVED BY MEMBERS OF THE THESIS COMMITTEE:

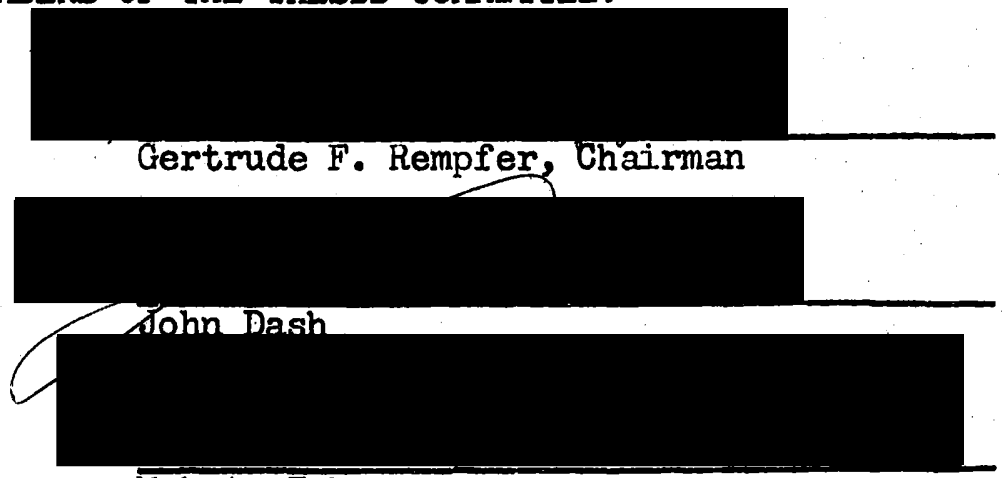

Makoto Takeo

The energy distribution in an electron beam transmitted by a thin aluminum film was studied by a retarding potential method in which the energy analysis could be made as a function of the polar angle and azimuth of scattering.

The results indicated that the transmitted beam contained electrons which had lost essentially zero energy and groups of electrons which had suffered losses of approximately $15 \mathrm{ev}$ and $25 \mathrm{ev}$, and possibly $75 \mathrm{ev}$. In addition there appeared to be a continuous background of enerey losses over the range studied (from 0 to $80 \mathrm{ev}$ ). The resolution of the energy analyser was limited by the separation of the data points and was not adequate to observe the dependence of the $15 \mathrm{ev}$ energy loss (plasma loss) on the polar angle of scattering. 
This study demonstrated the feasibility of this method of energy analysis as well as the short-comings of the apparatus. Suggestions for future improvements are made. 
A METHOD OF MEASURING THE ELECTRON ENERGY

LOSSES IN TRANSMISSION THROUGH THIN FILMS

by

JOHN PATRICK STEWART

A thesis submitted in partial fulfillment of the requirements for the degree of

MASTER OF SCIENCE

in

PHYSICS

Portland State University

1969 
TO THE OFFICE OF GRADUATE STUDIES:

The members of the Committee approve the thesis of John Patrick Stewart presented June 24, 1968.

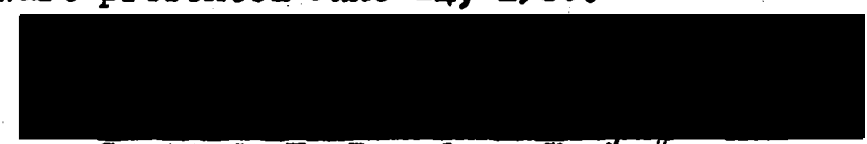

Gertrude F. Rempfer, Chairman

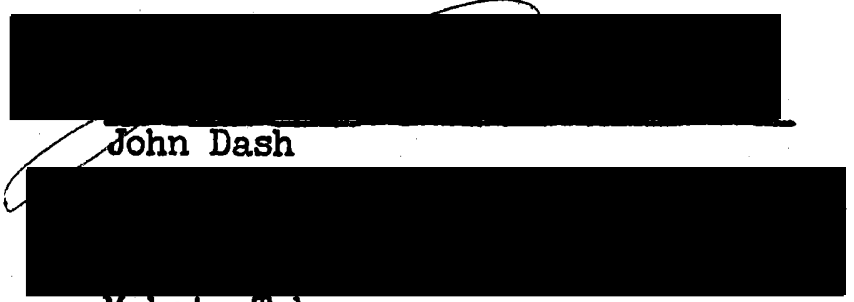

Makoto lakeo

APPROVED:

Mark Gurevitch, Head, Department of Physics

Frederick J. Cox, Dean (9i Graduate Studies

May 23, 1969 
TABLE OF CONTENTS

PAGE

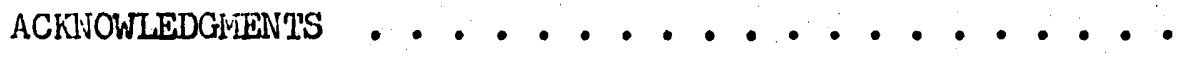

LIST OF TABLES . . . . . . . . . . . .

LIST OF FIGURES

$\checkmark$

CHAPTER

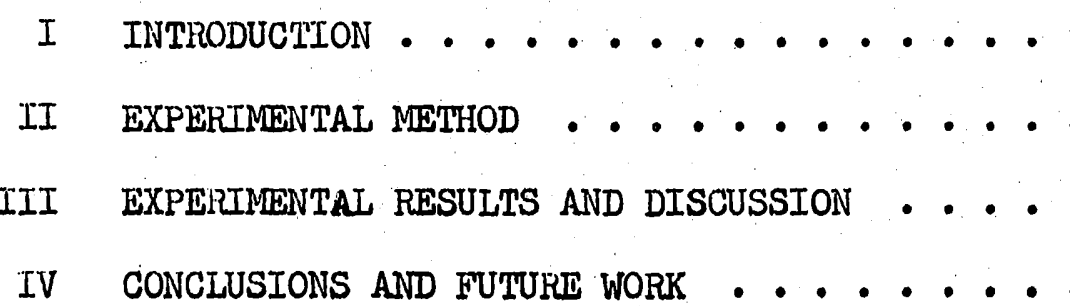

1

5

21

33

BIBLIOGRAPHY . . . . . . . . . . . 


\section{ACKNOWLEDGMENTS}

I wou.ld like to thank the members of my thesis committee, Professors Gertrude Rempfer, Makoto Takeo, and John Dash.

I am especially grateful to Professor Rempfer for the many hours she spent introducing me to experimental electron optics and to experimental physics in general. Professor Takeo was extremely helpful in reading and discussing several theoretical papers relating to this thesis. Mr. Michael Mauck was also helpful in general discussions about the apparatus. Mr. Jack Janacek, Head of the Science Division Machine Shop, and his assistant, Mr. Frank Bell, performed the machine work on the Apparatus. Mrs. Lorriane Mercer and Mrs. Laura Lusk of the Electron Microscope Group, Tektronix, Inc., prepared the specimens used.

I would like to express gratitude to my stepfather and mother, Mr. and Mrs. Wayland Byrd, for the financial aid given to my family and me during this study. Finally, I would like to express my gratitude to my wife, Mrs. Janice Stewart, for the technical assistance I received in preparing my thesis. 
LIST OF TABLES

TABLE

PAGE

I Essential Dimensions $\quad . . \cdot \cdot \cdot \cdot \cdot \cdot \cdot \cdot \cdot \cdot$ 
1 The focusing of scattered rays in the plane P. .

$2 \times 3.6$ enlargement of the diffraction pattern of aluminum taken with a specimen to plate distance of $18.2 \mathrm{~cm}$. and a primary beam voltage of $15 \mathrm{kv}$.

3 Schematic diagram of the analyser ...... 9

4 Electron-optical aspects of the analyser ... 11

5 Scattering pattern for aluminum taken at -5 volts bias ...........

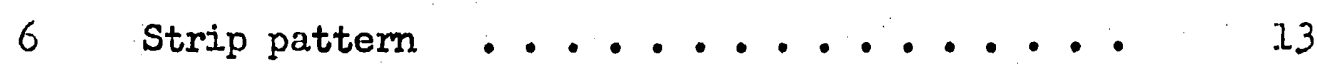

7 Pattern mask ................... 15

8 Schematic diagram of the experimental system . $\quad 17$

9. Physical set-up in the electron-optical bench . $\quad 18$

10 Essential Dimensions ............. 19

11 Calibration Curve: transmission of the primary beam by the analysing mesh as a function of the mesh bias voltage ..............

12--17 Transmission through the mesh analyser of the electron beam scattered by aluminum as a function of mesh bias voltage ..... 27-- 32

Al Intersection of the plane $P$ by the cones of scattered rays from $S_{0}$ and $s$.........

A2 Lack of registration of the intersection points on the plane $P$ in the direction of the specimen points 
FTCURE

PAGE

B1 Enerey analyser .......... 44

B2 Equilivant optical system . . . . . . 44

B3 Electron-optics of the mesh analyser . . 46

Cl Inclination of the electron paths to the axis of the system .............. 49 
CHAPTER I

INTRODUCTION

The study of the inelastic collisions which occur when a beam of electrons traverses a material provides a method for the investigation of the properties of matter. The energy losses which the electrons suffer during scattering can be measured in the emerging beam and give information about the electronic energy levels in the material. Typically a spectrum of energy losses for a material contains several peaks corresponding to discrete losses and a general background indicating a continuous range of energy losses. Many investigators have utilized the energy loss method in studying the properties of various materials. Much of the energy loss data has been assembled in a review article by Niarton, Leder, and Mendlowitz 9 .

Two of the possible mechanisms which can be identified as causing electron energy losses in metals are, according to Farrell ${ }^{6}$ : a) one-electron transistions where an electron in the metal is raised in energy from a level in one band to an unoccupied level in another band and b) excitation of plasma oscillations, which are collective oscillations of the charge density in the electron sea of the motal. The intensity peaks in the energy 
loss spectrum corresponding to interband transistions are generally not as sharp as those due to plasma oscillations. Interband losses may range down to low energies, for example around $3 \mathrm{ev}$ for gold. It should be possible to correlate the interband losses with optical absorption bands where data are available. On the other hand, the collective oscillation loss peak is relatively sharp and larger than the smallest interband losses. This loss is not expected to be correlated with an optical absorption band, but rather with a minimum cut-off frequency for transmission of electromagnetic radiation through the metal. Ferrell's viow is that in every metal there will be at least one collective loss, of relatively large energy and intensity, and none to several one-electron losses of generally smaller energy and intensity. Bohm and Pines ${ }^{1,2,3,11}$ have shown theoretically that collective oscillations above a certain critical wavelength corresponding to a maximum value of the frequency can exist in the electron sea. Watanabe ${ }^{12}$ has shown and verified experimentally that the frequency of the oscillation increases with the scattering angle of the exciting electron. For small scattering angles $\theta$ the frequency can be expressed as:

$$
\omega=\omega_{\mathrm{p}}+4 \pi \boldsymbol{\gamma}_{\mathrm{E}} \boldsymbol{\theta}^{2} / \mathrm{h}
$$

where $E$ is the kinetic energy of the exciting electron, $w_{p}$ is the plasma frequency for zero angle, and $\gamma$ depends on the scattering material. For metals which approximate the free electron model

$$
p=\left(4 \pi n e^{2} / m\right)^{\frac{1}{2}} \text {, and } \gamma^{\lambda}=0.45
$$


A number of different types of energy analysers have been used to obtain the energy loss spectrum of the scattered electron beam. 'l'he most widely used at present is a high resolution instrument devised by Mollenstedt. It is based on the high offaxis chromatic aberration of an electrostatic lens and has a resolution capability of less than one electron-volt for primary electrons of $20 \mathrm{kev}$. Comprehensive descriptions of this type of instrument have been given by Milyutin and $\mathrm{KabanOv}^{10}$ and $\mathrm{Hahn}^{8}$ along with examples of its application to the energy analysis of electron beams. Using this type of analyser, Watanabe ${ }^{12}$ observed both collective and one-electron energy losses and verified experimentally the properties of the collective oscillations for metals such as aluminum which approximate the free electron model used by Bohm and Pines. Another type of high resolution instrument, described by Haberstroh ${ }^{7}$, is based on a retarding potential method and measures the energy loss spectrum, integrated over smail scattering angles.

In most of the experimental studies the primary beam voltage, defined by $\mathrm{grv}^{2}=e^{V} \mathrm{~B}$, has been in the range from five to $45 \mathrm{kv}$. The energy losses do not depend on the primary beam voltage, but are characteristic of the material and are sometimes called eigenlosses.

Wultiple scattering, which is inherent in reflection studies and in transmission through thick specimens, produces additional peaks in the spectrum and increases the diffuse background. These complications are aroided if the specimen is thin 
enough so that the intensity of the singly scattered component of the emerging beam is large relative to that of the multiply scattered component. Thin specimens are essential in studies of the losses as a function of scattering angle.

The apparatus described in this thesis was designed to measure the electron energy loss spectrum as a function of the scattering direction. The electron beam is analysed in a plane following the specimen and normal to the axis of the primary beam. The position of the electrons in the analysing plane is determined by the direction of scattering. The convergence of the primary beam is such that electrons scattered in the same azimuth through the same polar angle by different parts of the specimen are focused in the analysing plane. The energy analyser is of the retarding potential type, but differs from that of Haberstroh which analyses the energy loss spectrum integrated over scattering angle and azimuth. The measurement of the energy loss spectrum was carried through for aluminum at room temperature with a primary beam voltage of $15 \mathrm{kv}$. 
CHAPTTR II

\section{EXPERIMENTAL METHOD}

A discussion of the electron optical aspects of the method used in this study are facilitated by Figures 1 through 4. As shown in Figure 1, electrons diverge from an essentially point source and are redirected by a lens toward a focus in the plane, P. The image of the source, formed in the plane $P$, is demagnified and is of the order of $0.001^{\prime \prime}$ in diameter. When a specimen is placed as shown in the path of the beam, rays which are scattered in a given azimuth through the same polar angle $\theta$ by different parts of the specimen are also focused, to first order, in the plane $P$ (see Appendix A). The energy loss analysis of the electron beam is carried out in the plane $P$ where the spectrum obtained as a function of position in the pattern can be correlated with the polar angle and the azimath of scattering. The diffraction pattern of the specimen is focused in this plane also. The contrast and sharpness of the diffraction pattern is an indication of the predominance of the single-collision components of the transmitted beam. Figure 2 is the diffraction pattern of the aluminum specimen used in this experiment. The dotted circle surrounding the center of the pattern included the range of angles over which the angular dependence of the energy Iosses was studied. 


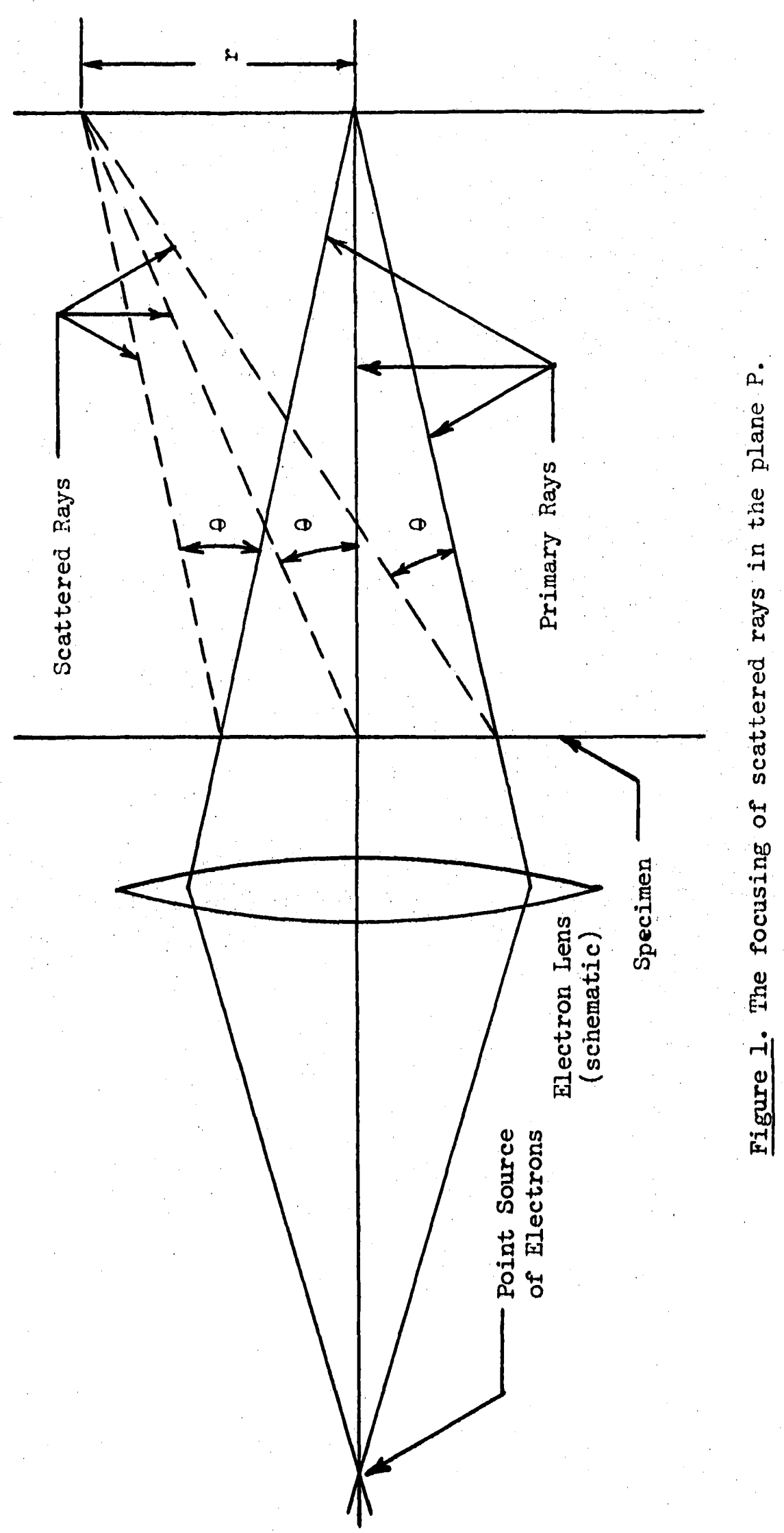




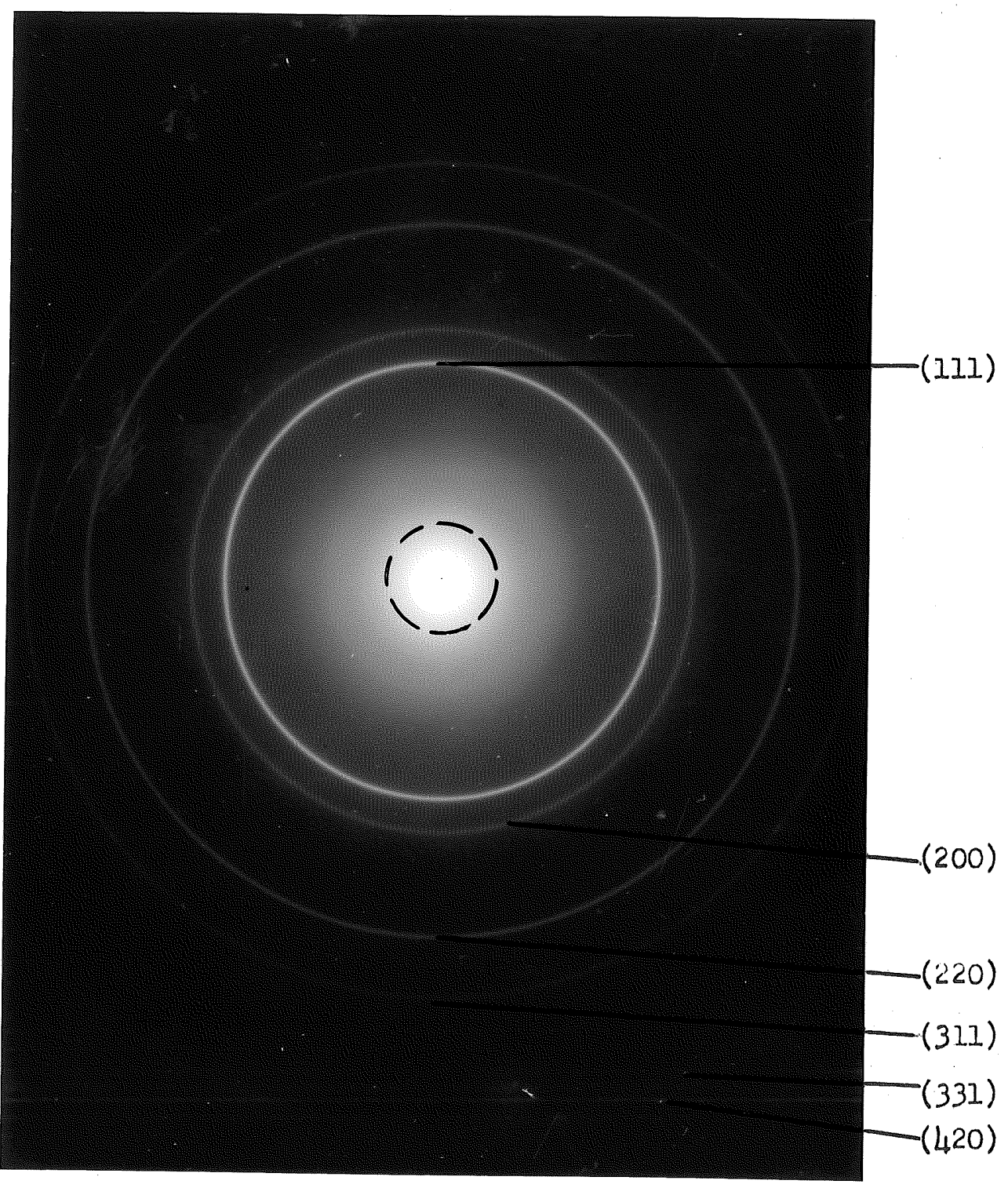

Figure 2. $\times 3.6$ enlargement of the diffraction pattern of aluminum taken with a specimen to plate distance of $18.2 \mathrm{~cm}$. and a primary beam voltage of $15 \mathrm{kv}$. 
The analyser consists of a fine metallic mesh supported between and electrically insulated from two outer electrodes which, along with the structure supporting the mesh, have cylindrical symmetry. The two outer electrodes are at ground potential as is the specimen, and the inner mesh electrode is negative and close to cathode potential (see the schematic diagram in Figure 3). An adjustable bias between the mesh and cathode potentials is used to analyse the distribution in energy of electrons in the beam. The mesh acts like a sieve allowing electrons above a certain energy (depending on the bias) to pass through while returning those of lower energy. Because of the field between the negative mesh and the grounded and electrodes the potential in the mesh openings is less negative than that of the mesh. Therefore a negative bias on the mesh is needed to cut-off transmission of the beam entirely. The changes in intensity of the beam transmitted by the mesh as the negative bias is reduced from cut-off gives the energy distribution in the beam.

Electron-optical effects in the analyser due to the electric fields between the electrodes and around the apertures in the end electrodes cause the effective position and size of the mesh, referred to the space outside the analyser, to be different from its actual position and size. The calculations are worked out in Appendix B. The result is that the mesh has a virtual magnification of $2 / 3$, and its distance from the aperture on the side of the reference space appears to be increased 


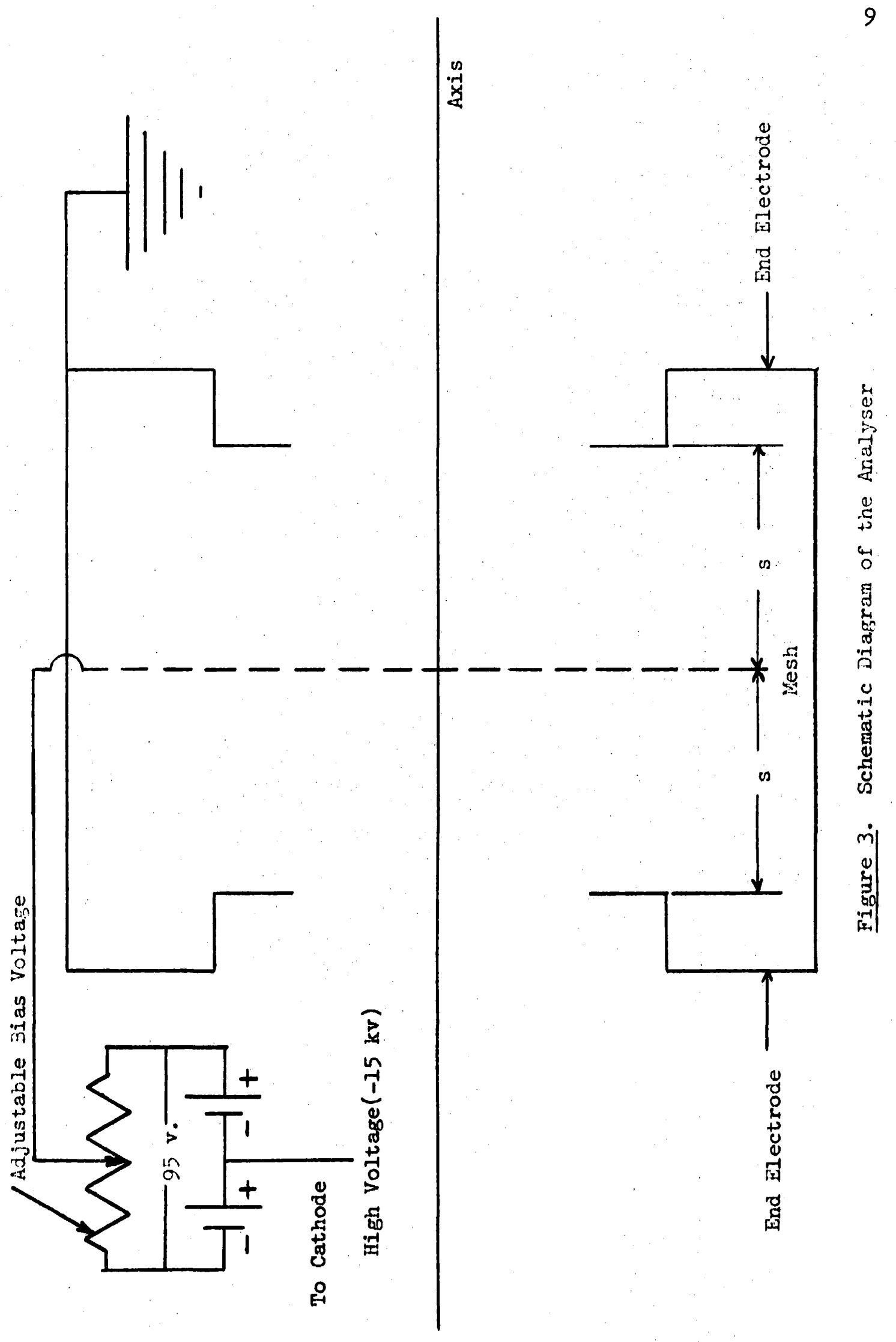


by the factor $4 / 3$ (see Figure 4). The plane $P$ in Figure 1 in which the scattering pattern is focused and in which the energy analysis is to be done should then be made to coincide with the virtual plane $M$ rather than $M_{0}$ in Figure 4 . Distances $r$ from the axis in this plane are measured in terms of the mesh constant of $M$ which is $2 / 3$ the size of $M_{0}$. The polar angle of scattering, $\theta$, corresponding to a distance $r$ off-axis is given by:

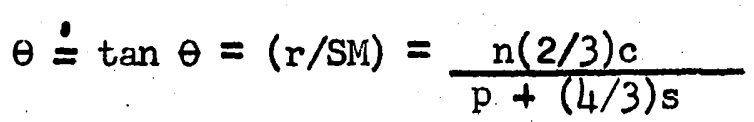

where $\mathrm{n}$ is the number of mesh spacings from the center and $\mathrm{c}$ is the mesh constant.

A further electron-optical aspect of the analyser is discussed in Appendix $C$. This involves the reduction in the axial speed of the electron as the scattering angle is increased. Correspondingly, the part of the beam voltage associated with the axial direction, defined by $\mathrm{QV}_{\mathrm{Z}}=(1 / 2) \mathrm{mv}_{\mathrm{z}}^{2}$, is reduced as the scattering angle is increased. For the chief ray of a diverging pencil, the loss in axial beam voltage of an electron inside the analyser is given by:

$$
V_{z}(10 s s)=V_{B} \theta^{2}(1+p / 4 s)^{2} \text {. }
$$

In general the beam voltage depends on both the scattering angle $\theta$ and the angle of convergence of, which results in a variation in axial beam roltage for a given pencil. The analysing mesh measures the axial energy and consequently records an apparant energy loss in the off-axis beam which must be taken into account in comparing the transmission through the 


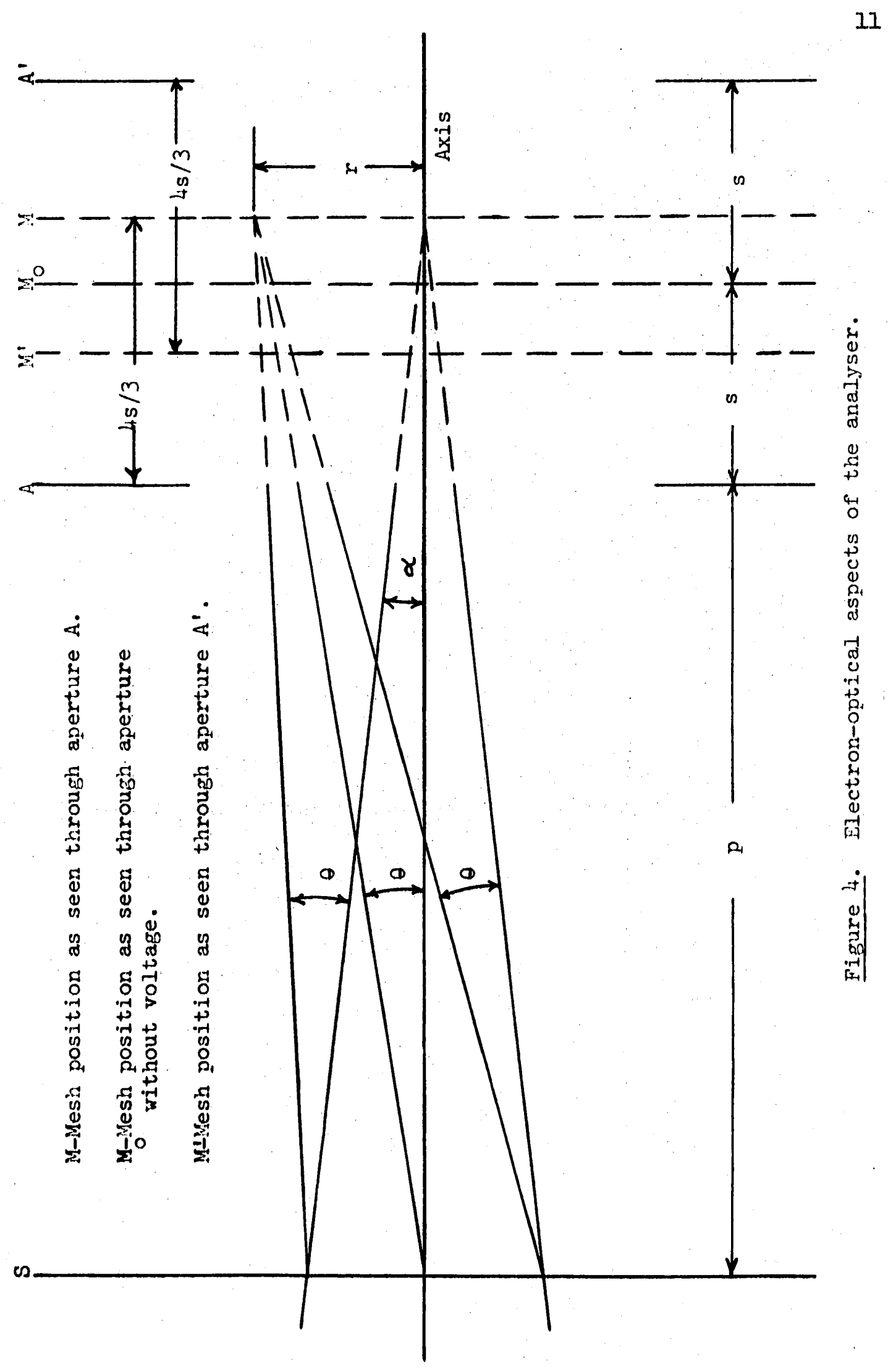


mesh of beams scattered at different angles. Furthernore the variation in axial beam voltage in a given pencil sets a limit on the energy resolution.

As the beam passes through the mesh it is scattered by the electric fields around the mesh openings, which act as an array of tiny lenses scrambling the rays from the different openings. The rays can be unscrambled by using an electron lens following the analyser to reimage the plane of the mesh. In addition, this lens can be used to magnify the image. This is desirable since the mesh spacing constant is very small $\left(0.002^{w}\right)$ and without magnification the analysis would be inconvenient. Ihe illumination in the mesh image as a function of position relates the current of the electrons getting through the mesh with the corresponding angle of scattering.

In the experimental arrangement, the magnified mesh image, illuminated by the electrons which the mesh transmits, can be observed on a fluorescent screen. The magnifying lens is adjusted so that the mesh is in focus and the specimen lens is adjusted so that the unscattered rays passing through the specimen are focused in the plane of the mesh. The mesh Image is then recorded on a photographic plate for study in a densitometer. Figure 5 is a sample photograph of the magnified image as recorded on the plate. The original intention was to record a full pattern for each mesh bias setting on a separate plate and from these plates to determine the energy distribution as a function of both azimuth and angle of scattering. However, the scattering 

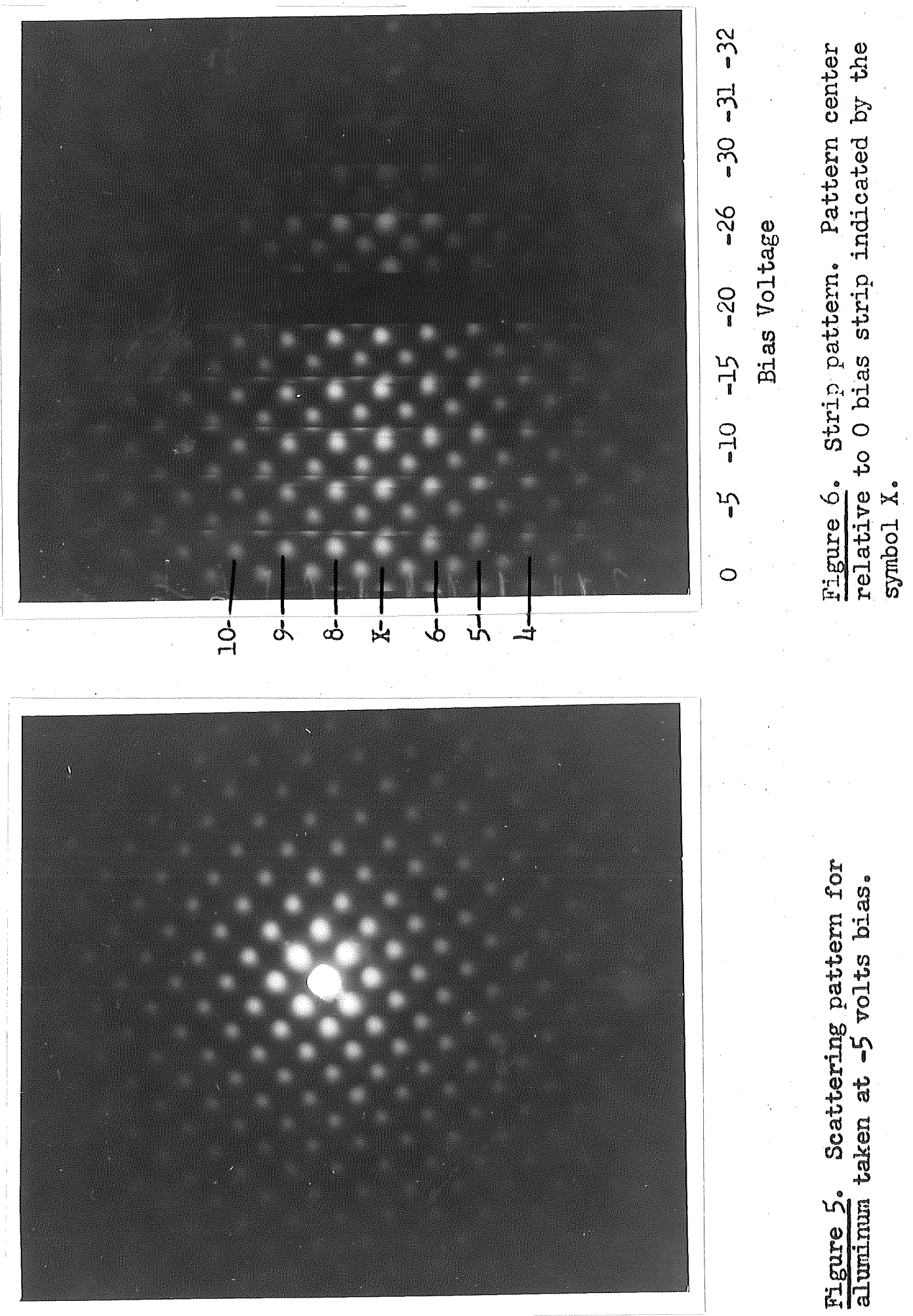

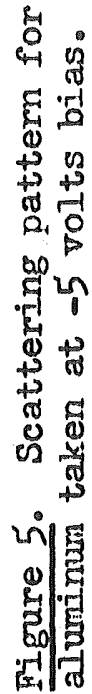


pattern for the specimen used appeared to be azimuthally symmetric and it was decided to record only a representative strip of the pattern. This made it possible to record patterns for a range of mesh bias settings on the same plate by advancing the plate behind a slit opening. The pattern strip was recorded for a series of mesh bias settings through the bias voltage range, -35 to +60 volts, and back. Four plates were needed for the series, which was recorded in sections: $-35 \mathrm{v}$ to 0,0 to $+60 \mathrm{v}$, $+60 \mathrm{v}$ to 0 , and 0 to $-35 \mathrm{v}$. Figure 6 illustrates the appearance of the pattern strips for the range from zero bias to cut-off. The location of the center of the pattern relative to the strip is indicated in the sketch.

The plates were examined to determine the variation in density with mesh bias at a given position in the image. Densitometer readings for a given mesh opening were taken for the series of bias settings and plotted as a function of bias voltage. The effective image density for a mesh opening was obtained by masking off the surrounding patterm (see Figure 7) and measuring the average transmission of light through the mesh hole image. The effective density is related to the current of electrons through the mesh opening. A comparison of the density readings, corresponding to exposures taken at the same bias setting (each setting occurred twice in a series) served to check on individual differences in the plates and on progressive changes in bearn intensity, e.g., as might be caused by the heating current falling off in the emitting filament. The analyser was 


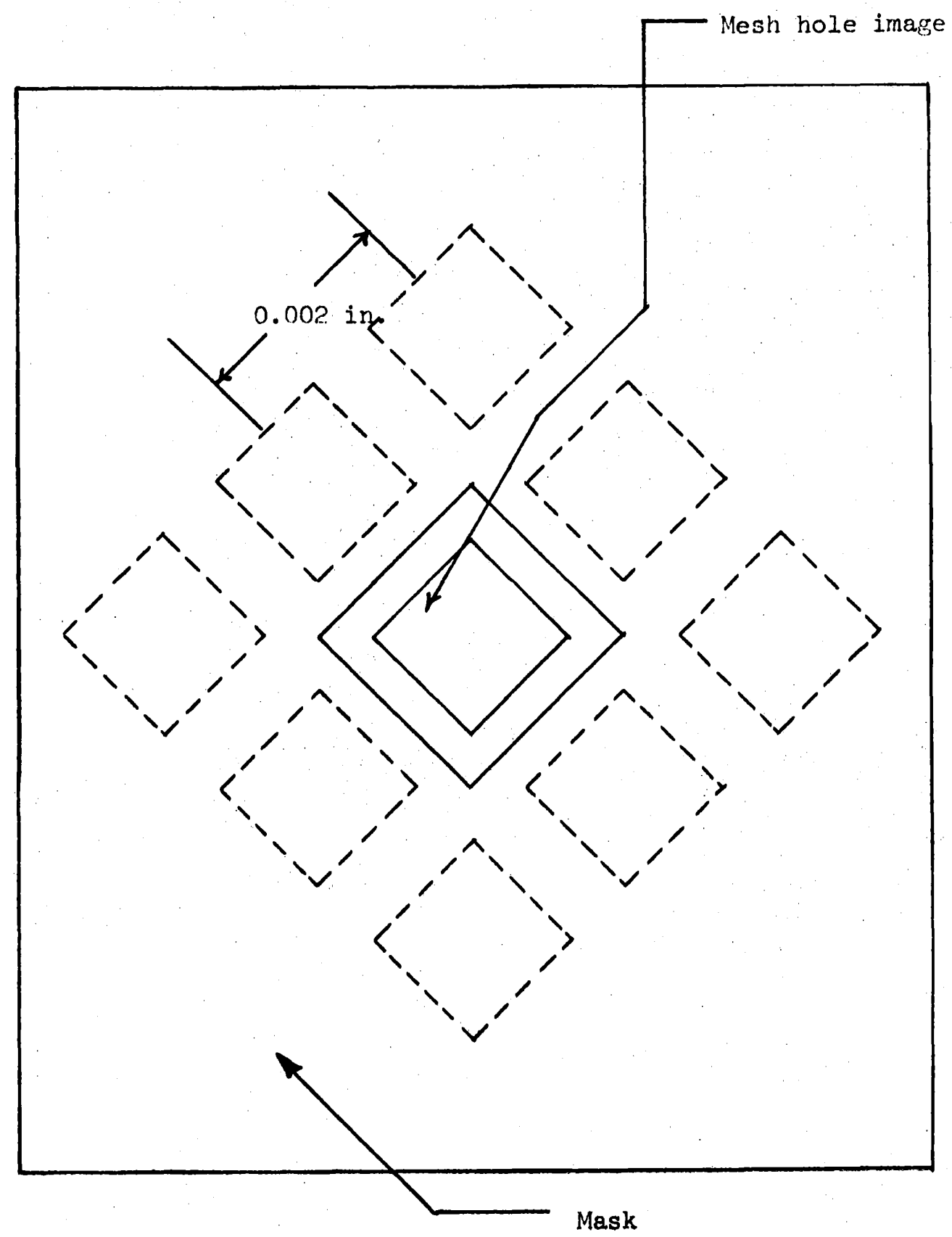

Figure 7. Pattern mask. 
calibrated by recording the transmission of the primary monoenergetic beam through the mesh (without a specimen) and plotting the density versus bias settings in the same way. The experiment was conducted in an electron-optical bench. The pressure was below $10^{-4}$ Torr. The bench was provided with V-ways to align the optical components of the spectrometer, electrical feedthroughs for supplying voltages, an electron source, and a multiple photographic plate holder. A glass top for viewing the experiment was removable for access to the system. The "point source" of electrons was formed by using a condenser lens to demagnify the waist of the beam from the electron source. The angular aperture $\alpha$ of the beam directed through the specimen was limited to about 0.007 radians by a stop on the specimen lens. The magnifying lens also was stopped down to avoid undue spherical aberration. These lenses were of the three-electrode unipotential type with a variable focal power depending on the ratio of the lens voltage to the accelerating voltage. The physical system was arranged so that the anode was at ground potential and the cathode negatively off ground. Figure 8 is a schematic diagram of the experimental system and Figure 9 is a photograph of the setup in the electron-optical bench. The table in Figure 10 gives the numerical value of the essential dimensions. 


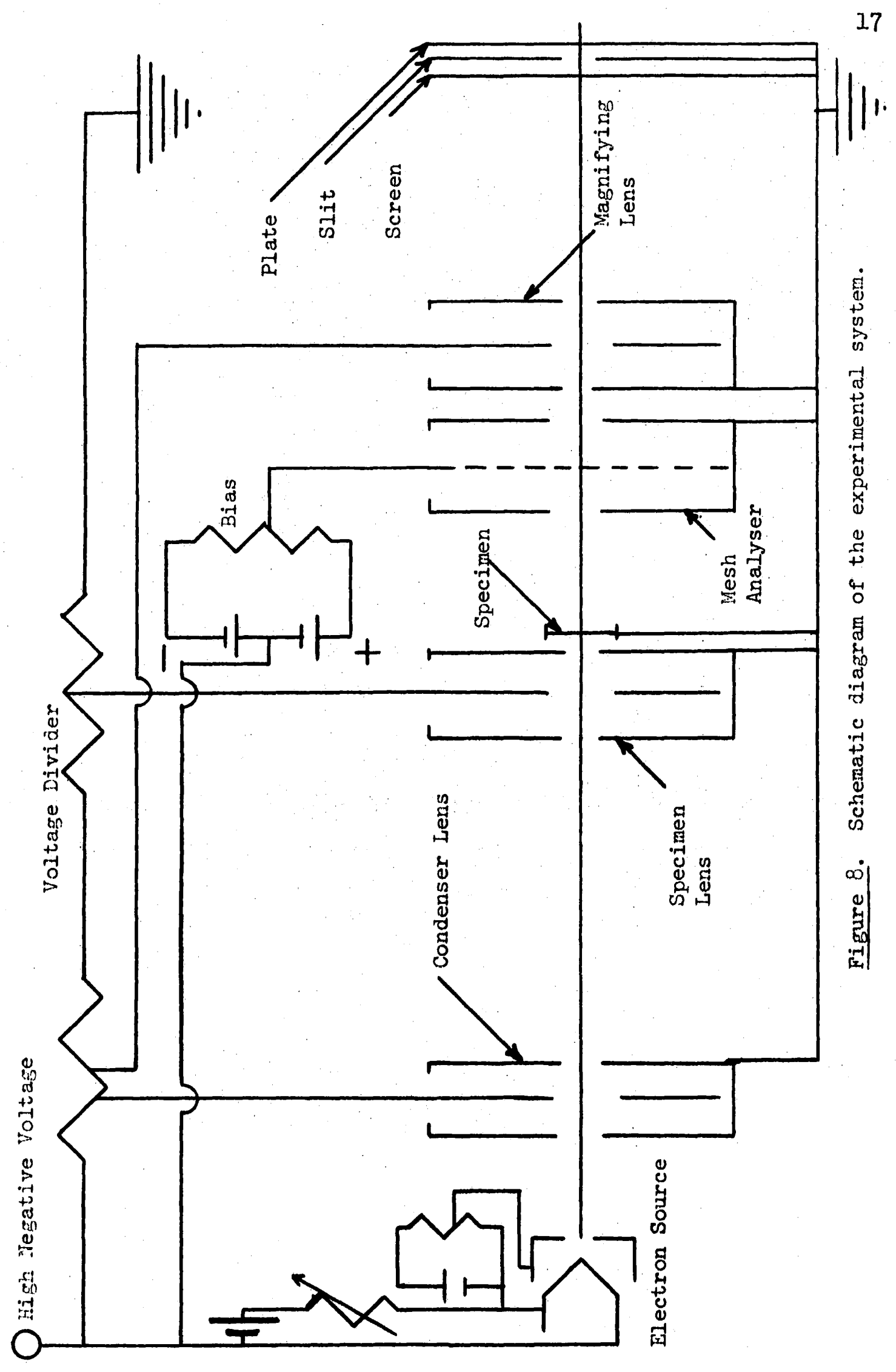




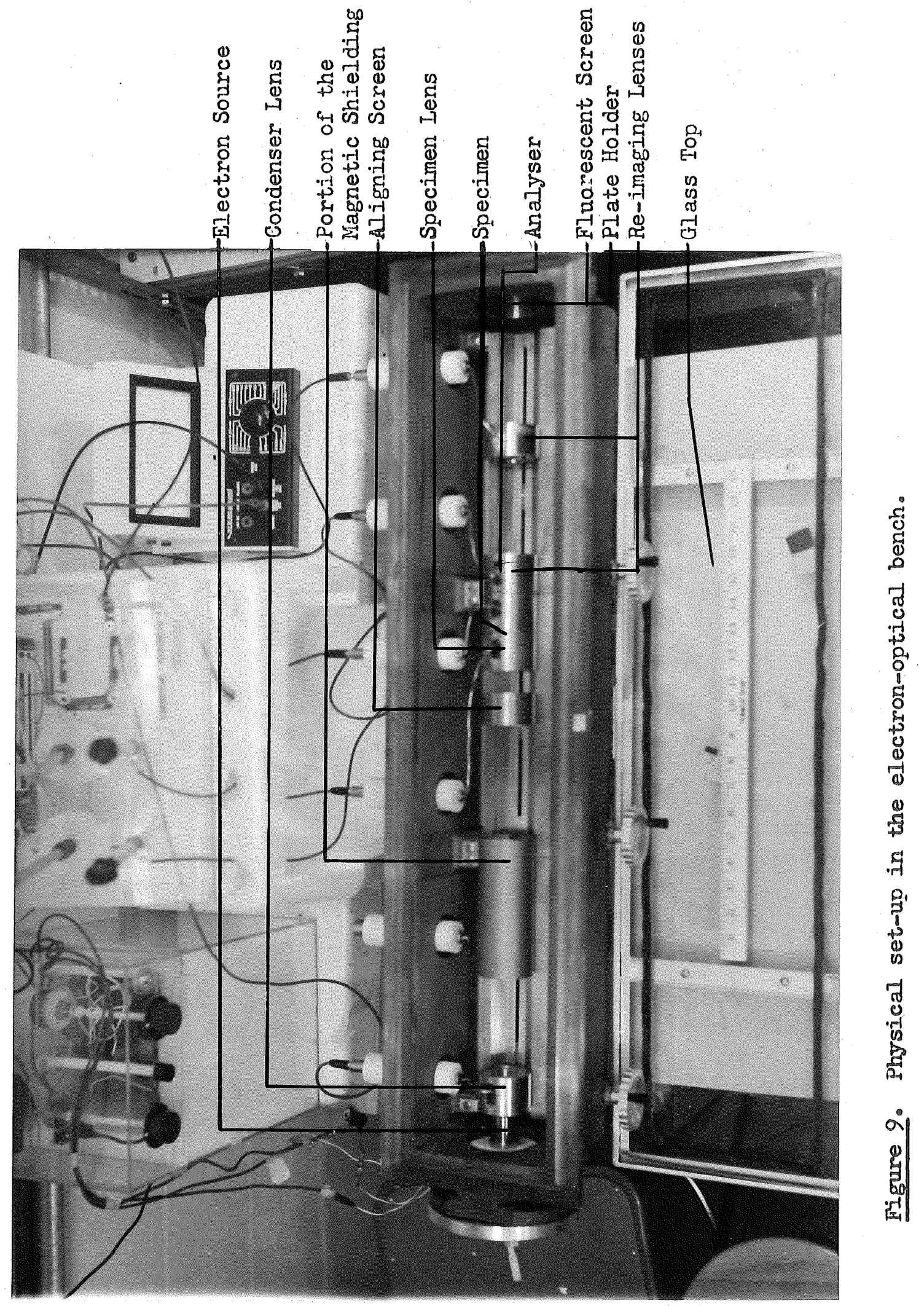




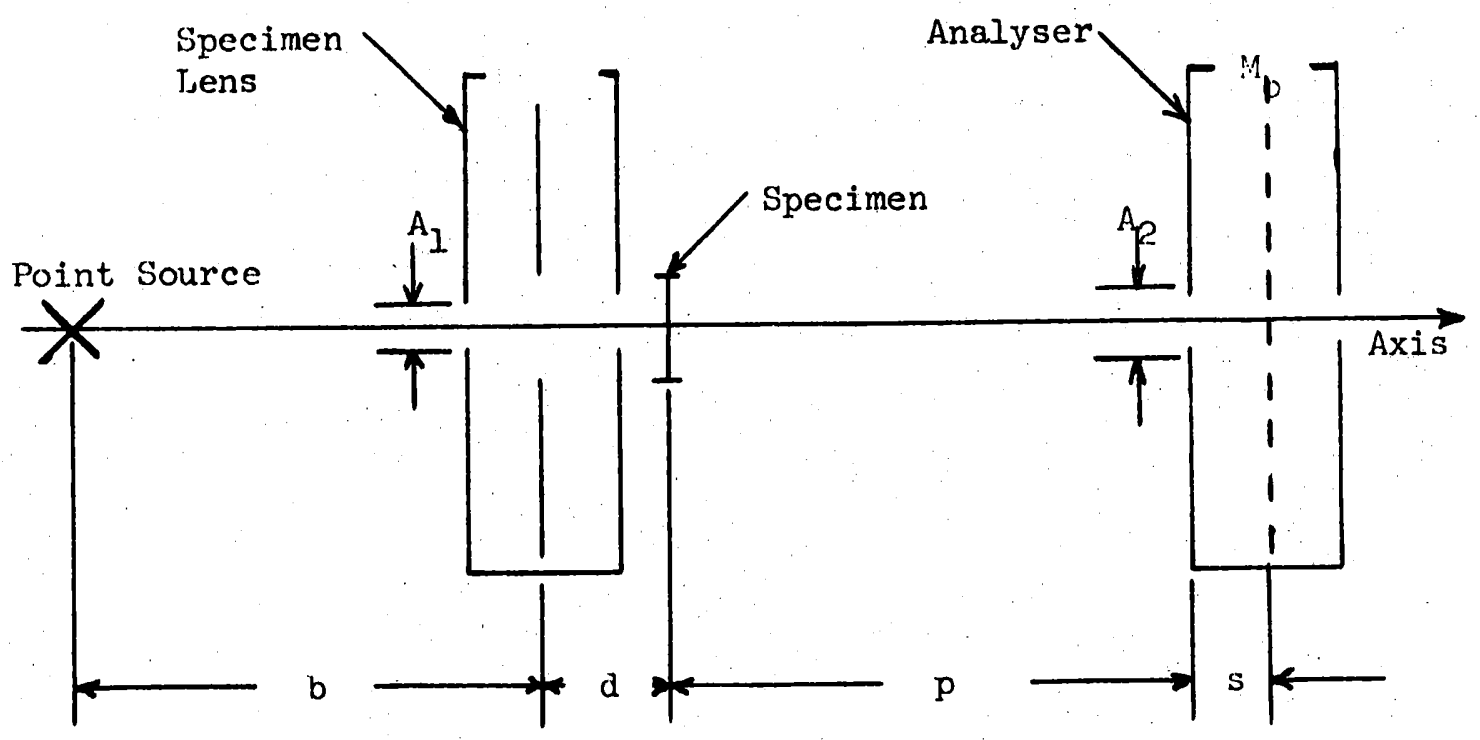

Figure 10. Schematic Diagram of the essential dimensions.

Table of dimensions

\begin{tabular}{cl}
\hline & Value \\
\hline $\mathrm{b}$ & $41 \mathrm{~cm}$. \\
$\mathrm{d}$ & $1.4 \mathrm{~cm}$. \\
$\mathrm{p}$ & $2.01 \mathrm{~cm}$. \\
$\mathrm{s}$ & $0.65 \mathrm{~cm}$. \\
$\mathrm{A}_{1}$ & $0.067 \mathrm{~cm}$. \\
$\mathrm{A}_{2}$ & $0.384 \mathrm{~cm}$.
\end{tabular}

Table I. Essential dimensions. 
The aluminum specimen, approximately 100 angstroms thick, was prepared by vacuum evaporation onto mica. It was subsequently floated off on water and mounted on a standard electron-microscope grid for study in the apparatus. 
CHAPTER III

EXPERIMENTAL RESULTS AND DISCUSSION

The experimental data are represented graphically in Figures 11 through 17. Each figure is a plot of effective image density as a function of bias voltage from -35 volts to +60 volts. The density scale and unit are arbitrarily chosen so that the numerical value is zero at cut-off and one at zero bias. The density readings going toward and coming from cut-off are shown as separate points, $(O)$ and $(O)$, at the ends of the vertical bars.

Figure 11 is the calibration curve for the mesh, taken without the specimen. The beam angle $\theta$ was zero. The density curve drops sharply to zero as cut-off (at -33 volts) is approached and indicates a resolution of one volt at the toe. A sharper cut-off could be obtained with a finer mesh in the analyser and finer steps in the bias adjustment. A slight spread in the axial beam voltage and consequently a olight unsharpness in cut-off is caused by the convergence of the beam heading toward focus in the analysing plane. The angle of convergence, after the divergent effect of the aperture field of the analyser is included, is about 0.005 radians. This causes a spread in axial beam voltage for the unscattered rays of less than one-half volt which is below the present resolving power. 
The "sagging" of the potential across the mesh openings accounts for the cut-off occurring at -33 volts instead of zero bias. Figures 12 through 17 are image density versus bias voltage plots for electrons transmitted by the thin aluminum specimen at room temperature for scattering angles of 0.0024 , 0.0035 , and 0.0055 radians. The two curves for each angular setting are for two different mesh openings on opposite sides of the center of the strip. The insert is the density plot from cut-off to zero bias with an expanded vertical scale. All of the curves show a sharp cut-off at about -32 volts. This is very close to the cut-off on the calibration curve at $\theta=0$ and corresponds to a group of electrons which have lost no appreciable energy. A comparison of the curves for beams scattered at different angles indicates a slight shift in the cut-off bias over the angular range. The shift of about one volt is consistent with the shift to be expected because of loss in "axial beam voltage" for beams inclined to the axis (see Appendix $($ ). The spread in axial beam voltage due to the convergence of pencils inclined to the axis sets a resolution limit of about two volts at the largest scattering angle for which measurements were taken. The spread in axial beam voltage can be reduced by the use of a smaller stop to decrease the angular aperture of the converging pencil.

Another abrupt rise in the density as the bias increases positively from cut-off occurs between -20 and -15 volts (in all of the curves except that in Figure 14). This rise occurs at 
about 15 volts from cut-off and can be accounted for by a group of electrons which have lost $15 \mathrm{ev}$ of energy and which begin to be transmitted as the mesh potential becomes 15 volts more positive than cut-off. The $15 \mathrm{ev}$ Ioss is approximately equal to the $14.8 \mathrm{ev}$ loss found by Watanabe and which he attributed to the excitation of a plasma or collective oscillation. Over the range of scattering anglies used, this energy loss was expected to show a shift of about one volt in the position of the toe as a function of scattering angle. However, the bias voltage steps were not fine enough (except near cut-off) to accurately determine the location of the toe of the density rises. It was therefore not possible to verify the angular dependence.

Most of the curves show a density rise occurring at a bias voltage between -10 and -5 volts corresponding to an energy loss of approximately $25 \mathrm{ev}$. This is close to the $23 \mathrm{ev}$ loss which Watanabe found and attributed to an interband transistion related to the fine structure in the $I$ absorption edge for $x$-rays. There is also a slight indication of a density rise at +45 volts mesh bias corresponding to a loss of about $75 \mathrm{ev}$. The $75 \mathrm{ev}$ loss is outside the range of losses studied by Watanabe, however a loss of approximately this amount has been related by Cauchois ${ }^{4}$ to the fine structure in the $K$ absorption edge for $x$-rays.

A continuous background distribution of electron energies is suggested by the general slope of the specimen curves, which could be due to radiation losses which occur when the electron 
is accelerated in collisions. The general slope may be due in part also to small intensity interband losses which were not observed because of lack of resolution. The data assembled by Marton, Leder, and Mendlowitz ${ }^{9}$ include the losses observed in this study along with others not observed here.

The inserts on some of the figures show the separate image density curves for increasing and decreasing bias from cut-off to zero. The differences between the curves of increasing and decreasing bias may be due to differences in the plates used to record these sections of the series of bias voltage settings. It is also possible that the beam had changed during the run, but the method of choosing the density scale for the curves would tend to minimize this effect. However, in both curves the same energy groups appear, as indicated by the position of the steeper slopes, although not with the same amplitude. In Figures 12 and 13 where only one curve appears the photographic data for one direction of bias change was impaired by fogging.

The density versus bias curves in Figures 12 and 18 are for scattering angles of 0.0024 radians. Two separate mesh openings, Nos. 6 and 8 , on opposite sides of center were measured to obtain these curves. In Figure 12 the energy losses are more noticeable than in Figure 18 but have the same values. The differences between the curves may be duo to differences in the scattered beam in the two scattering azimuths which 
would indicate that the scattering was not azimuthally

symmetric as supposed earlier.

Figures 14 and 15 are for scattering angles of 0.0035

radians corresponding to mesh openings Nos. 5 and 9. The curve in Figure 14 differs from all of the other specimen curves in that the energy losses appeared to be shifted toward cut-off by about 5 volts. At present, the explanation for this is not known.

The curves in Figures 16 and 17 are for scattering angles of 0.0055 radians and correspond to mesh openings Nos. 4 and 10. Again, the energy losses appear to be the same but have different intensities. Again the only explanation is that the scattering pattern was not azimuthally symmetric. 


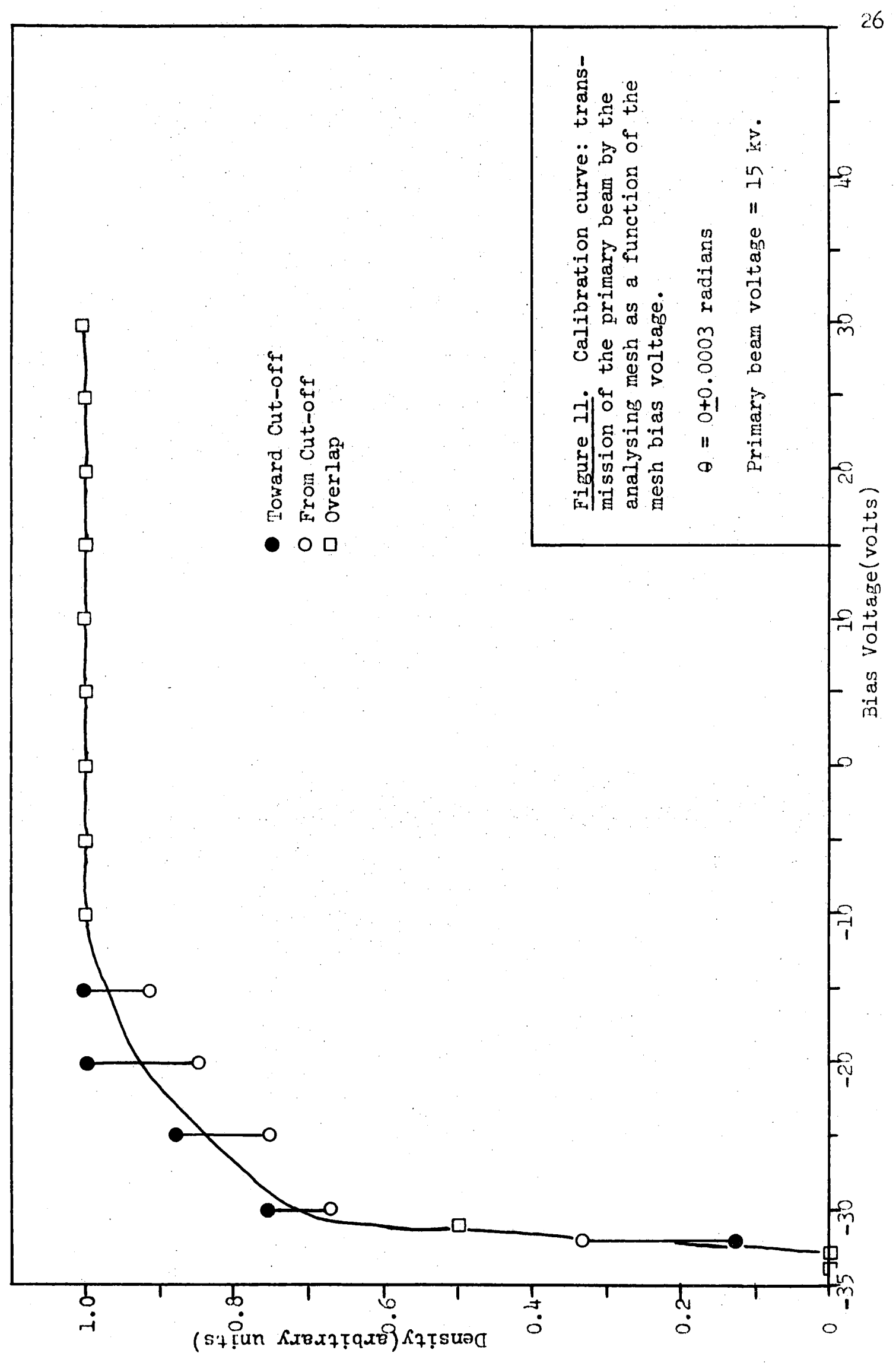




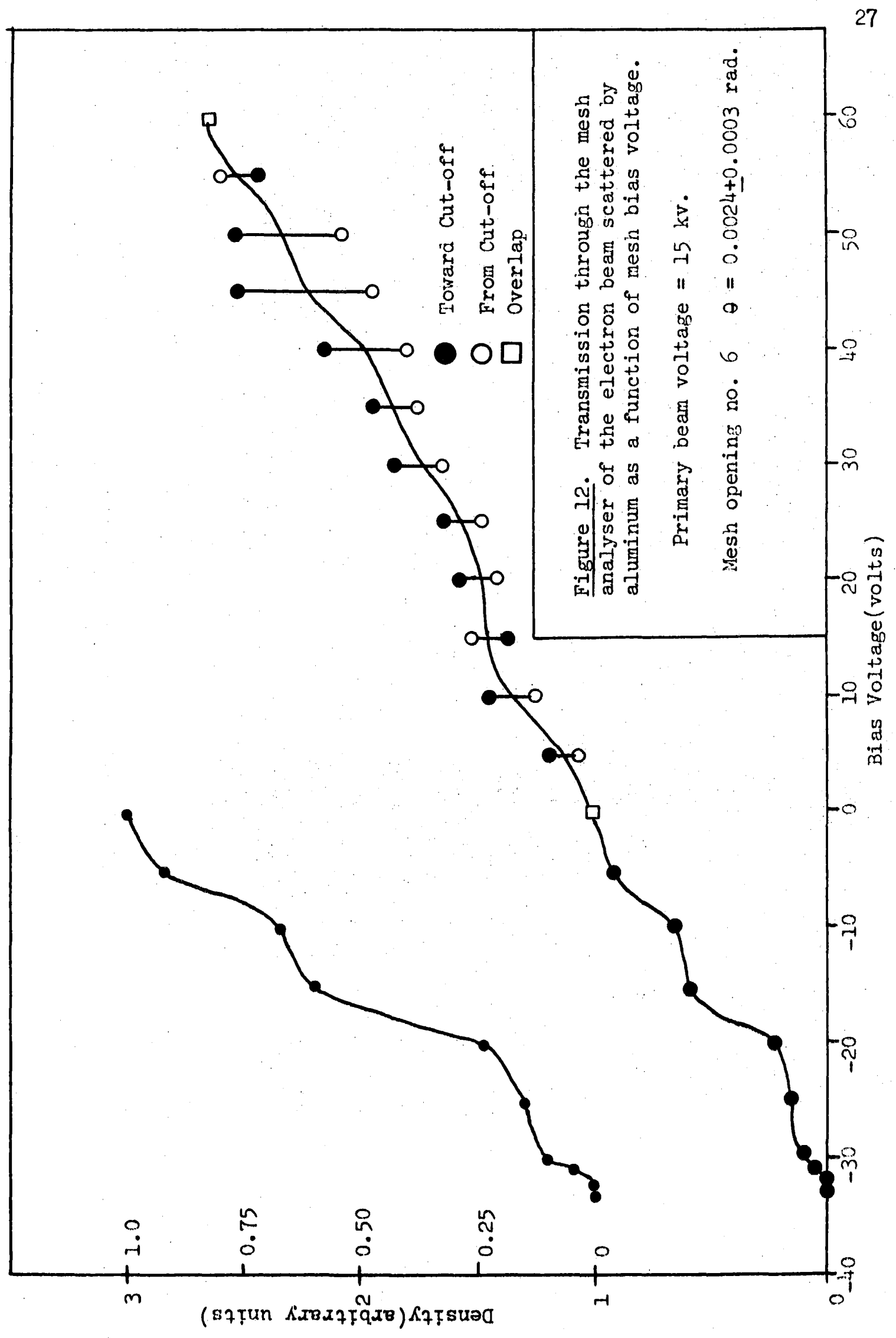




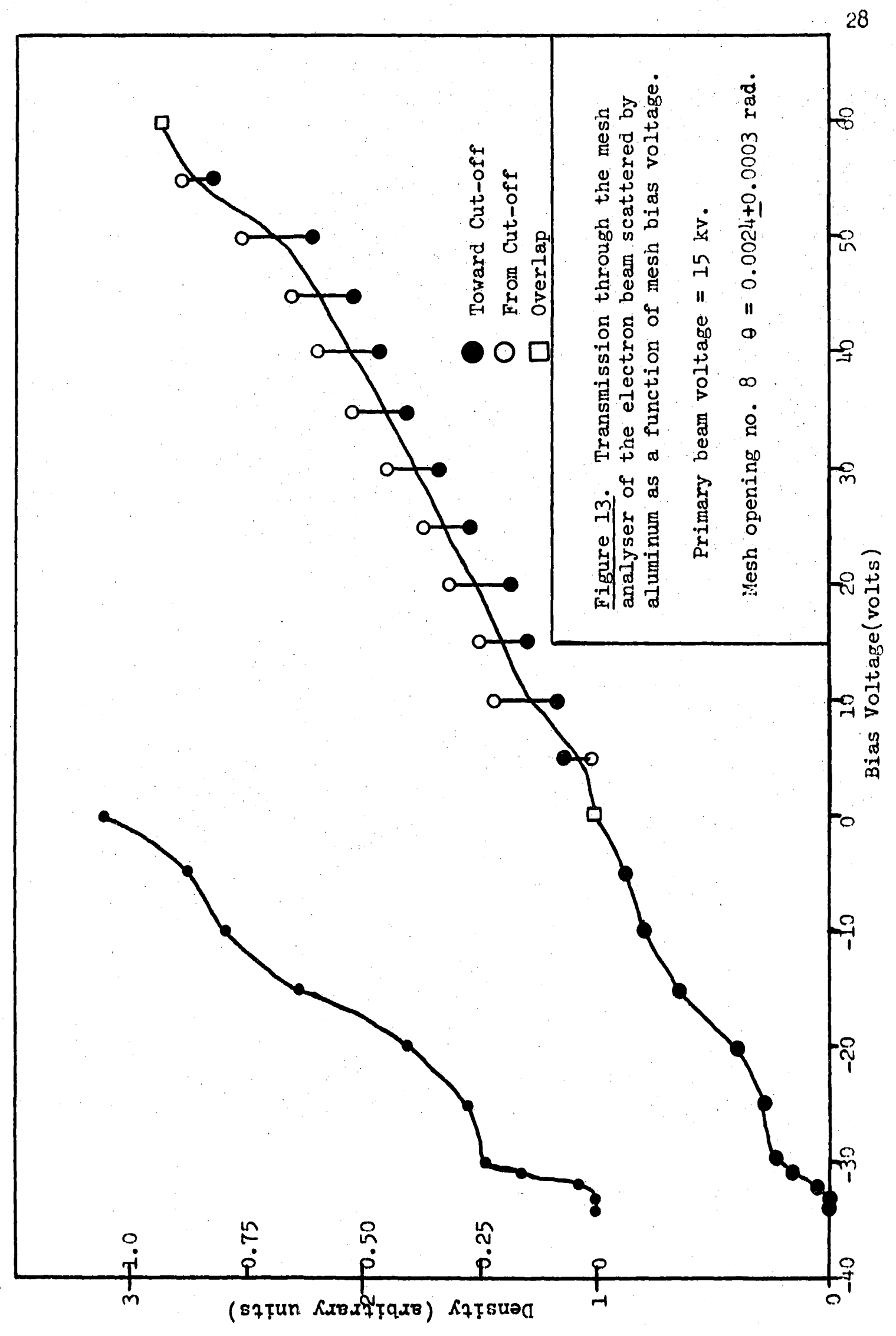




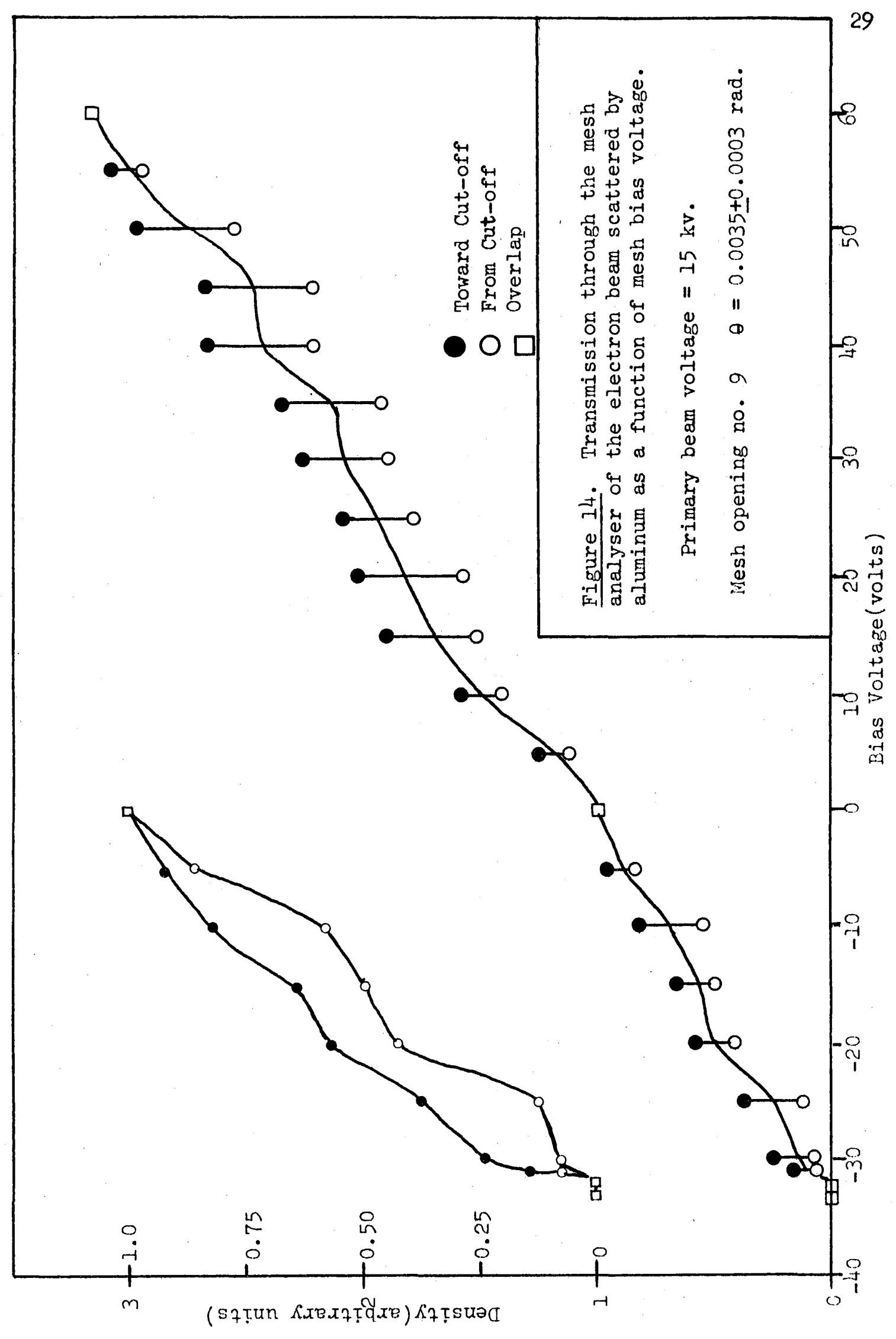




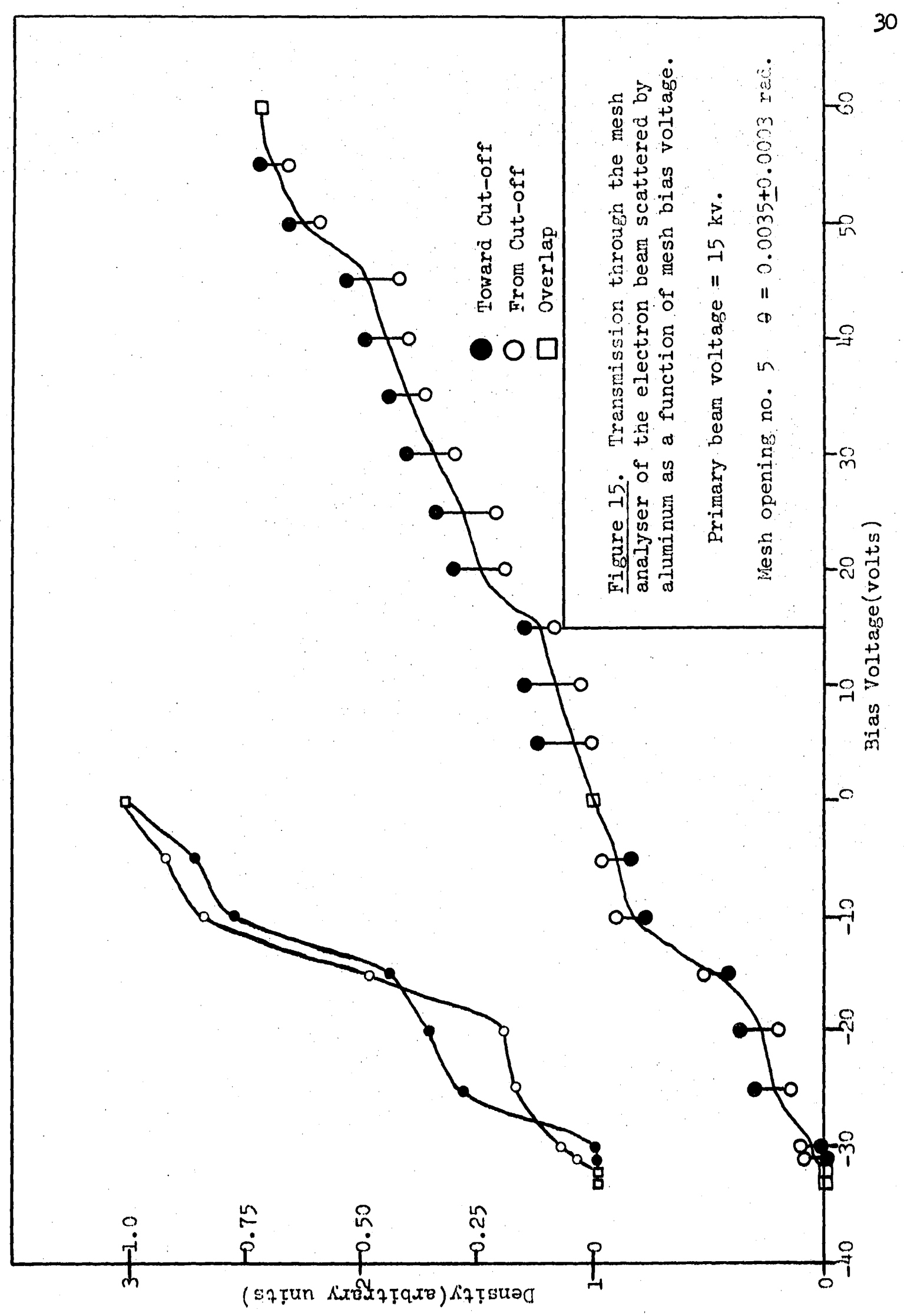




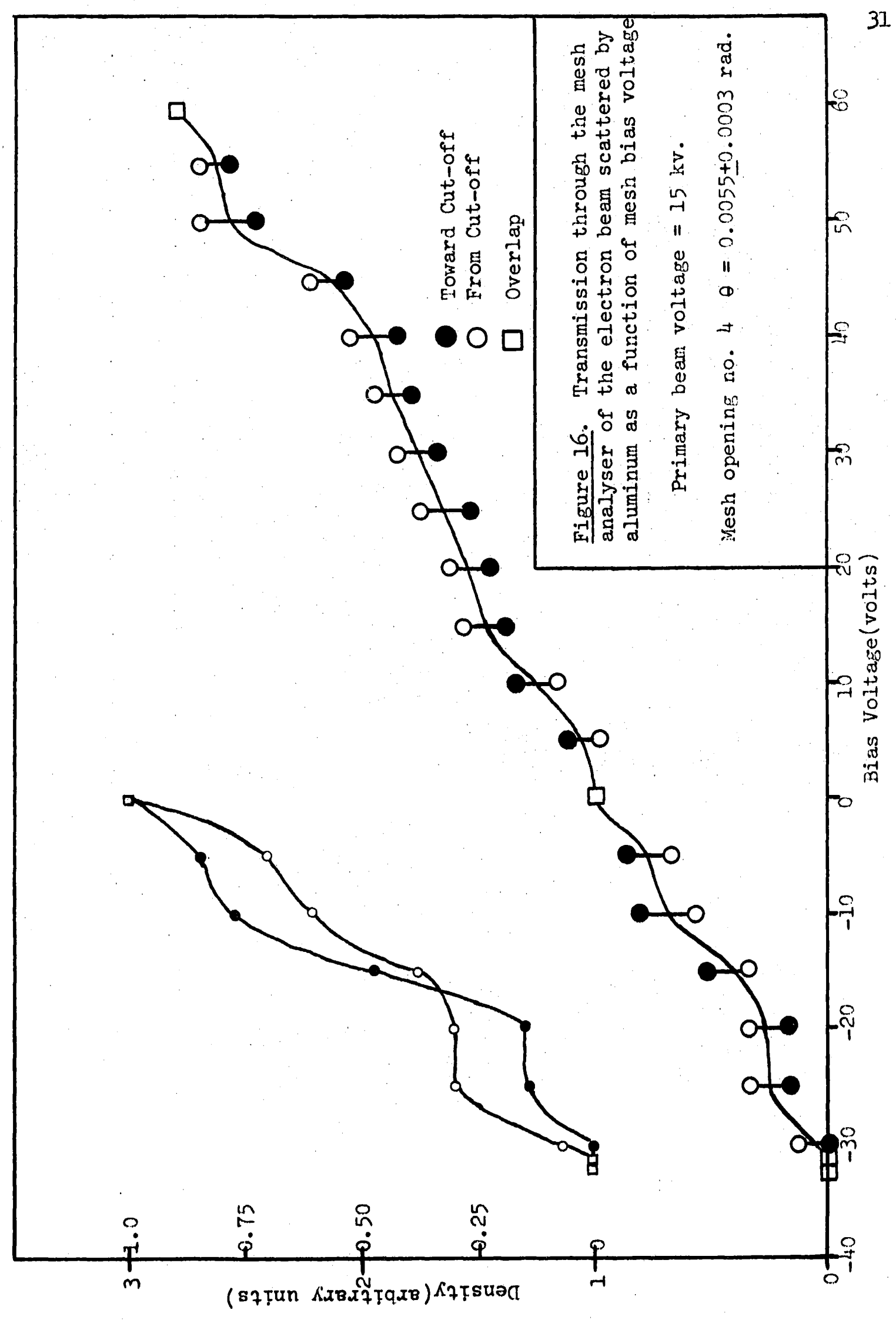




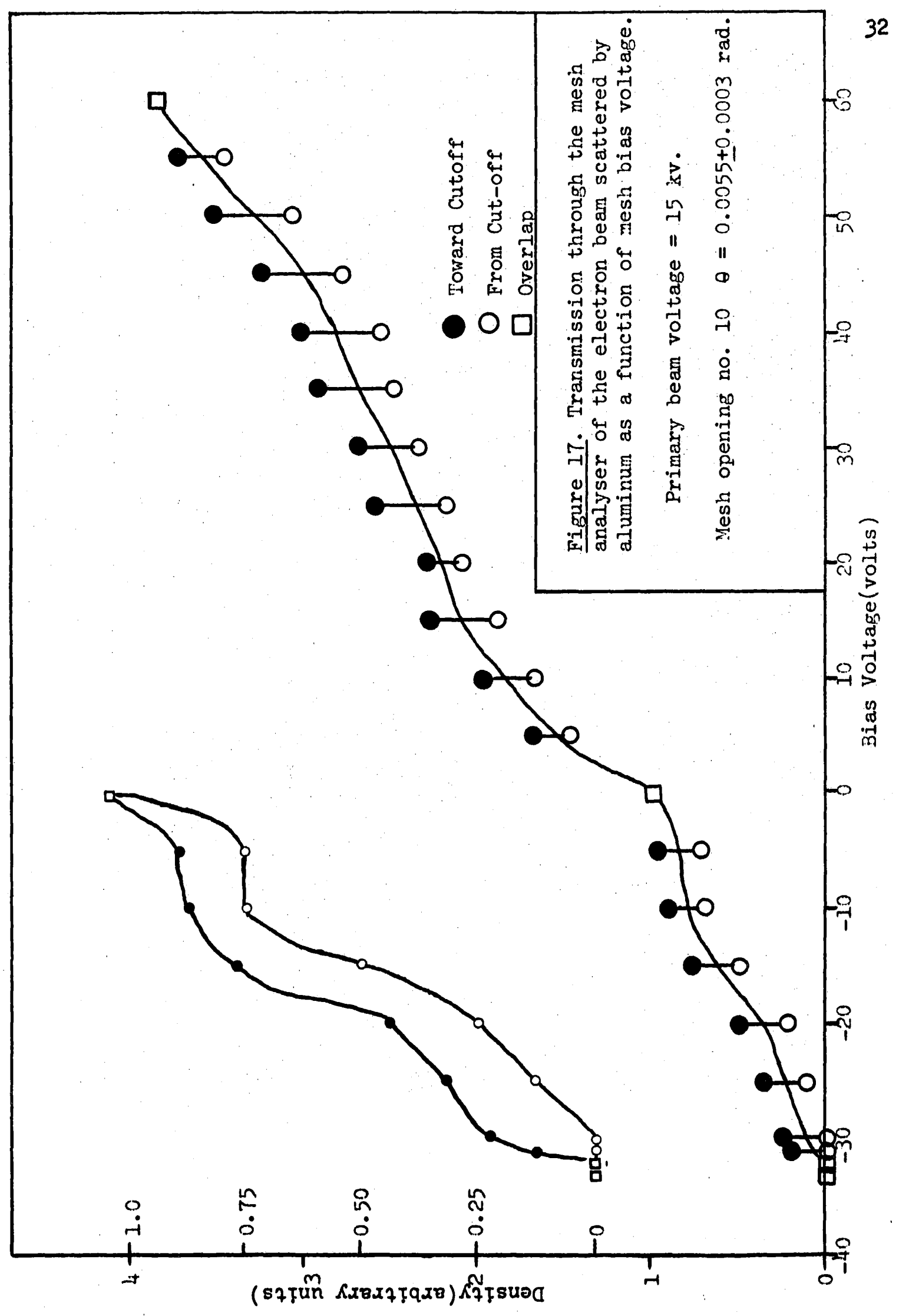


CHAPTER IV

\section{CONCLUSIONS AND FUTURE WORK}

The energy distribution in an electron beam, transinitted by a thin aluminum film, which served as a test specimen, was studied by a retarding potential method in which the energy analysis could be made as a function of the polar angle and azimuth of scattering. The primary energy was $15 \mathrm{kev}$. The results showed that the transmitted beam contained electrons which had lost essentially zero energy along with groups of electrons which had suffered losses of about $15 \mathrm{ev}$ and $25 \mathrm{ev}$, and possibly $75 \mathrm{ev}$. In addition there appeared to be a continuous background of electron energy losses over the range studied (from 0 to $30 \mathrm{ev}$ ). The $15 \mathrm{ev}$ loss has been attributed to the excitation of plasma or collective oscillations of the electrons in the specimen, and the 25 and 75 ev losses have been attributed to interband transitions. The continuous background could be due to radiation losses, and unresolved smal. intensity interband losses. It was not possible to observe the dependence of the plasma loss on the angle of scattering because of lack of resolution in the mesh bias settings. This study demonstrated the feasibility of this method of energy analysis of electron beams as well as the shortcomings of the apparatus and measurements. 
The resolution of the energy analysis could be improved considerably by finer settings in the bias voltage. However, taking and working up the data would be very time consuming if the photographic method described in this thesis were used. It would seem better to use a different method of recording the image obtained; e.g., a fluorescent screen and a photomultiplier tube could be used to measure the illumination at a given position in the pattern as the bias voltage is varied continuously through its range. If, in addition, the bias voltage and photomultiplier outputs were used to run an XY-plotter, data recording would be greatly facilitated. Another improvement in resolution would result from using a finer mesh in the analyser. Meshes of 2000 lines per inch are available commercially, whereas the mesh used in this experiment was 500 lines per inch.

The axial beam voltage loss due to angle of scattering can be avoided by arranging the specimen lens between the specimen and the analyser in such a way that the pencils of scattered rays have their axes normal to the analysing mesh inside the analyser. In this arrangement the individual pencils of scattered rays converge, as before, toward a focus in the plane $\mathrm{Ni}$ at a distance $4 \mathrm{~s} / 3$ beyond $A$. However, the specimen is positioned so that its image is projected at a distance $4 \mathrm{~s}$ beyond A. The chief rays of the scattered pencils head toward the axial point of the image and on entering the analyser are diverged by the aperture lens into a parallel beam normal to the analysing mesh. 
A smaller angle of convergence of the pencils of rays

focused in the analysing plane would reduce the spread in axial beam voltage resulting from this cause. The angle can be decreased by the use of a smaller stop on the specimen lens or by an increase in distance between the specimen and the analysing plane.

It would be interesting to apply this method, with improvements of resolution, to other specimens. It would be especially interesting to study a case where the scattering pattern is not rotationally symmetric. 


\section{BIBLTOGRAPHY}

1. Bohm, D. and Pines, D. "A Collective Description of Electron Interactions: I. Magnetic Interactions." Physical Review, 82: 625-634. 1951.

2. Bohm, D. and Pines, D. "A Collective Description of Electron Interactions: II. Collective vs Individual Particle Aspects of the Interactions." Physical Review, 86: 338-353. 1952.

3. Bohm, D. and Pines, D. "A Collective Description of Electron Interactions: III. Coulomb Interactions in a Degenerate Gas." Physical Review, 92: 609625. 1953.

4. Cauchois, P. Y. "Etude compare's du Spectra K d'Absorption de l'Aluminium Me'tallique." Acta Crystallographie, 5: 351-356. 1952

5. Davisson, C. J. and Calbick, C. J. "Electron Lenses." Physical Review, 42: 580. 1932

6. Ferrell, R. A. "Angular Dependence of the Characteristic Energy Ioss of Electrons Passing Through Thin Foils." Physical Review, 101: 554-563. 1956.

7. Haberstroh, G. "Untersuchung der charakteristischen Energieverluste mit Hilfe der Gegenfeldmethode." Zeitschrift fur Physik, Bd. 145: 20-53. 1956.

8. Hahn, E. "Zur Theorie des elecktrostatischen Geschwindigkeitsfilters." Jenaer Jahrbuch, 1961/II. 325.

9. Marton, I., Leder, L., and Mendlowitz, H. "Characteristic Energy Losses of Electrons in Solids." Advances in Electronics, 7: 183-238. 1955. 
10. Milyutin, V. I. and Kabanov, A. N. "An Electrostatic Electron-Velocity Analyser." Advances in Physical Sciences, IXI: 588-624. 1957 (Translated from Uspekhi Fizicheskikh Nauk).

11. Pines, D. "A Collective Description of Electron Interactions: IV. Electron Interaction in Metals." Physical Review, 92: 626-636. 1953

12. Watanabe, H. "Experimental Evidence for the Collective Nature of the Characteristic Energy Ioss of Electrons in Solids-Studies on the Dispersion Relation of Plasma Frequency." Journal of the Physical Society of Japan, 11: 112-119. 1956. 
APPENDICES 
APPENDIX A

FOCUSING OF SCATIERED RAYS

In Figure Al rays scattered by two different specimen points, $S_{0}$ and $S_{1}$, into cones of the same angle $\theta$ are shown. The axes of these cones are inclined to each other at an angle $\alpha$ and intersect at $\theta$ in the plane P. The cone of rays from So has its axis normal to $P$ and intersects $P$ in a circle of radius $z \tan \theta$. The azimuth of the scattered ray corresponds to the angle $\emptyset$ in the plane. The rays from $S_{1}$ intersect $P$ in an ellipse. The points of intersection do not coincide perfectly with those from so either in distance from 0 or in azimuth in the plane. The lack of registration of the intersection points is greatest in the direction of the separation of the specimen points. The rays corresponding to this direction are drawn in Figure $A 2$. These rays from $S_{I}$ intersect $P$ at $r_{\max }$ and $r_{\min }$. From the law of sines:

$$
\frac{r_{\max }}{\sin \theta}=\frac{2 \sec \alpha}{\sin \left(90^{\circ}-\theta-\alpha\right)}=\frac{2 \sec \alpha}{\cos (\theta+\alpha)}
$$

or

$$
r_{\max }=\frac{2 \sin \theta \sec \alpha}{\cos (\theta+\alpha)}
$$

Also from the law of sines:

$$
\frac{r_{\min }}{\sin \theta}=\frac{2 \sec \alpha}{\sin \left(90^{\circ}-\theta+\alpha\right)}=\frac{2 \sec \alpha}{\cos (\theta-\alpha)}
$$




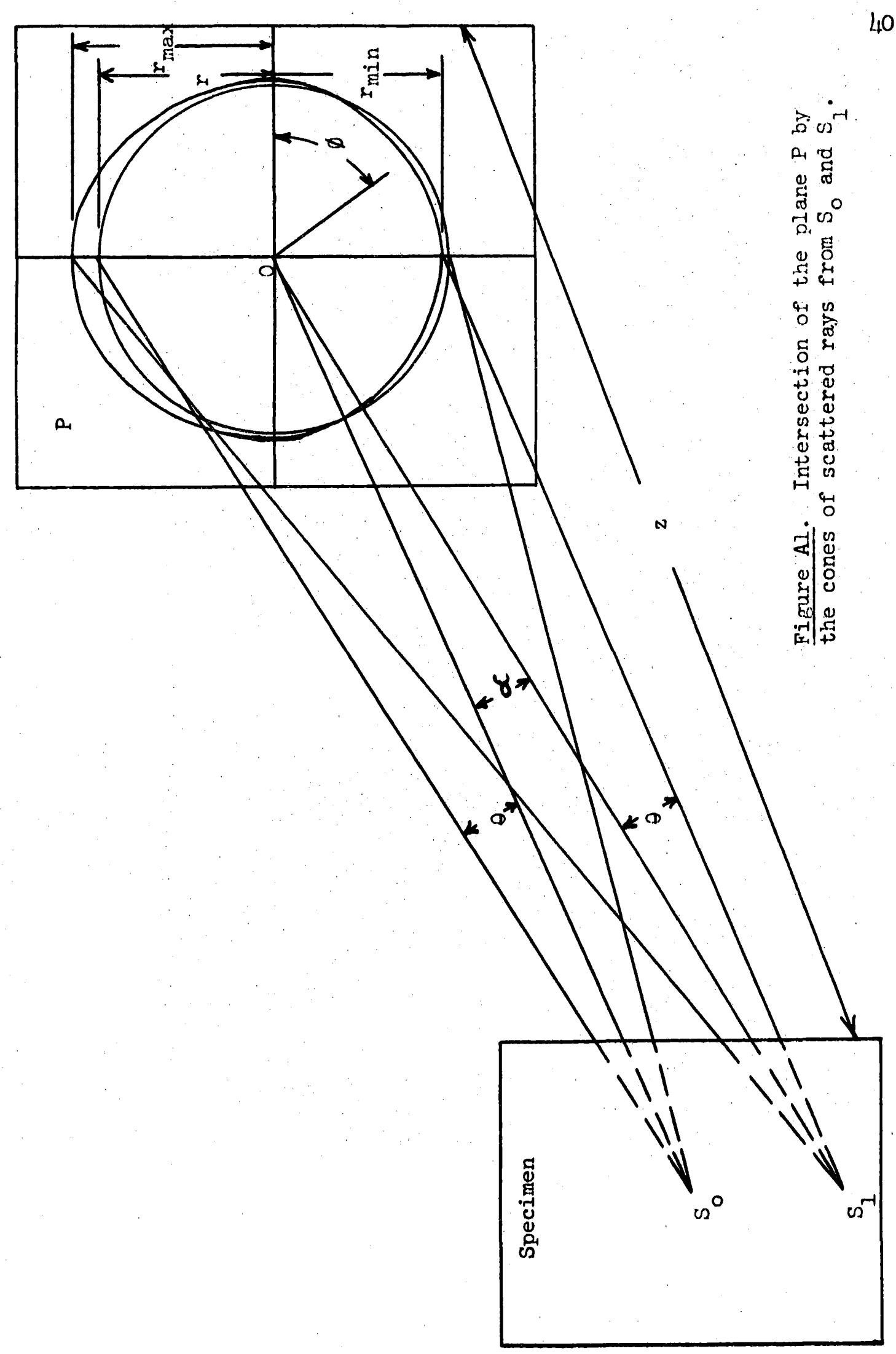




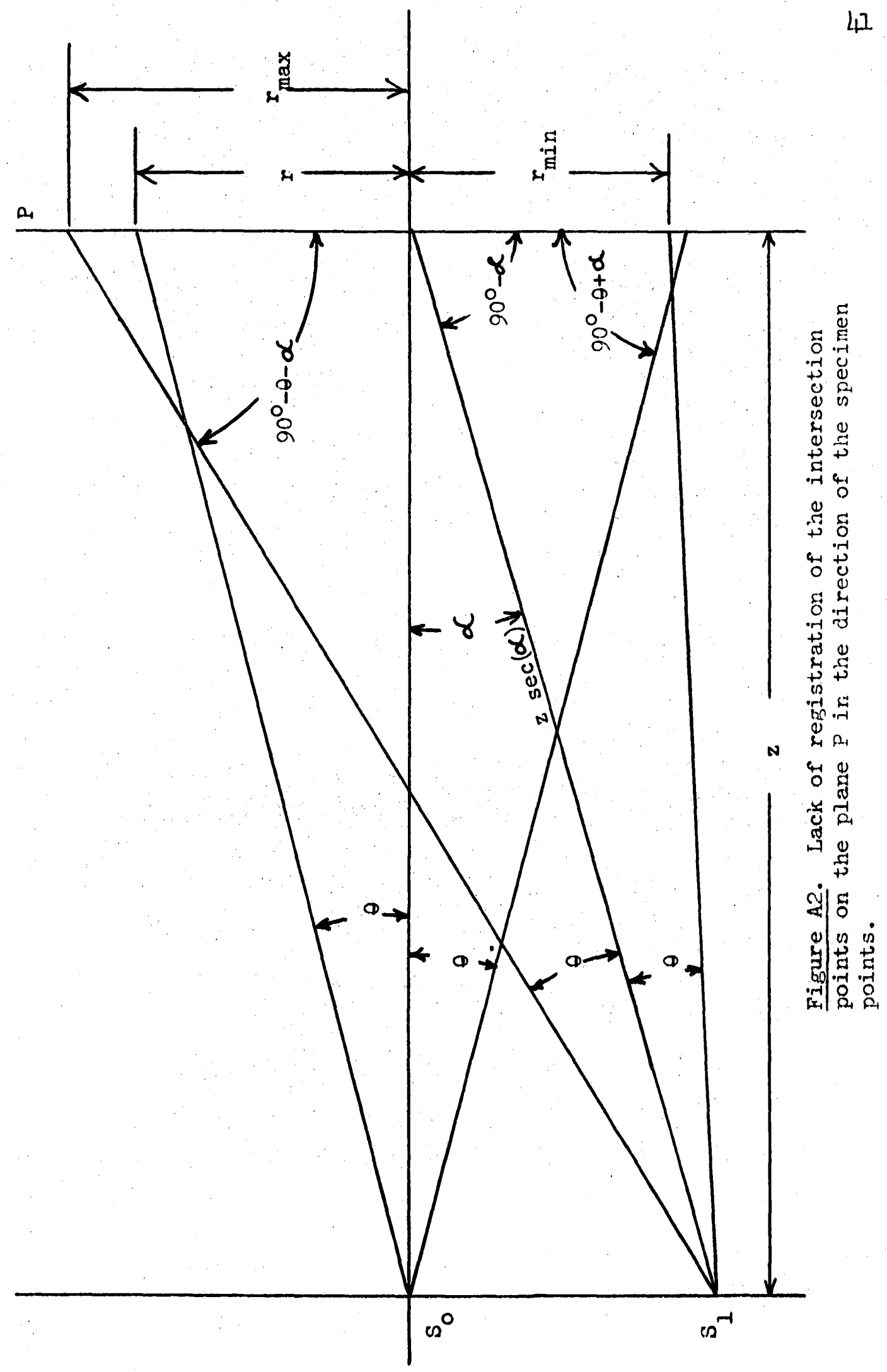


or

$$
r_{\min }=\frac{2 \sin \theta \sec \alpha}{\cos (\theta-\alpha)}
$$

The rays from $S_{0}$ intersect $P$ at:

$$
\mathbf{r}=\mathbf{z} \tan \theta
$$

The expressions for $r_{\max }, r_{\min }$, and $r$ differ only in second and higher order terms in $\theta$ and $\alpha$. For $\alpha=0.007$ radians and $\theta=0.006$ radians (the largest value of $\theta$ was 0.0055 radians), and $z=p+(4 / 3) s=2.88 \mathrm{~cm}, r_{\max }$ can be evaluated:

$$
\begin{aligned}
r_{\max } & =2 \theta\left(1-\theta^{2} / 3 !\right)\left(1+\alpha^{2} / 2 !\right)\left(1+(\theta+\alpha)^{2} / 2 !\right) \\
& =r\left(1-6 \times 10^{-6}+25 \times 10^{-6}+85 \times 10^{-6}\right) \\
& =r\left(1+10^{-4}\right) .
\end{aligned}
$$

The lack of registration for $r_{\min }$ and $r$ is less than that for $r_{\max }$ and $r$.

At a distance of $\sqrt{20}$ mesh spacings $\left(0.002^{\prime \prime}\right)$ for the largest $\theta$ used and for a mesh opening of 0.001 " the fractional variation of $r$ over the mesh opening is:

$$
\frac{0.001^{\prime \prime}}{(\sqrt{20}) 0.002^{\prime \prime}}=1 / 9
$$

which is much larger than the lack of registration ( $10^{-4}$ parts of $r$ ) of $r_{\max }$ on $r$. Therefore the ellipse and circle intersection curves can be considered to be in registration. The azimuths of the intersection points also agree to first order and can be considered to be in registration. 


\section{APPENDIX B}

\section{ELECTRON-OPTICAL ASPECTS OF THE ANALISER}

The equivalent optical system for the analyser is the same on both sides and is therefore worked out for one side only (see Figure BI). The effect of the electric field in the analyser can be thought of as the sum of the separate effects of two fields: A uniform field between the positive end electrode $A$ at potential $V_{A}$ and the negative mesh electrode $M_{0}$ at potential $V_{M}$, and a field associated with the aperture in the end electrode. The local fields associated with the small openings in the mesh are taken into account separately. The aperture field was shown by Davisson and Calbick 5 to be, to first order, a thin electron lens of focal length:

$$
f=\frac{4 v_{B}}{(\partial V / \partial z)_{2}-(\partial V / \partial z)_{1}}
$$

$V_{B}$ is the beam voltage of the electron at the potential of the apertured electrode and is equal to $V_{A}-V_{C}$ where $V_{C}$ is the cathode potential. $(\partial V / \partial z)_{2}$ and $(\partial V / \partial z)_{I}$ are the potential gradients which would exist on the two sides of the electrode in the absence of the aperture and have values of:

$$
(\partial V / \partial z)_{1}=0 \text { and }(\partial \nabla / \partial z)_{2}=\frac{\left(V_{A}-V_{M}\right)}{s}
$$



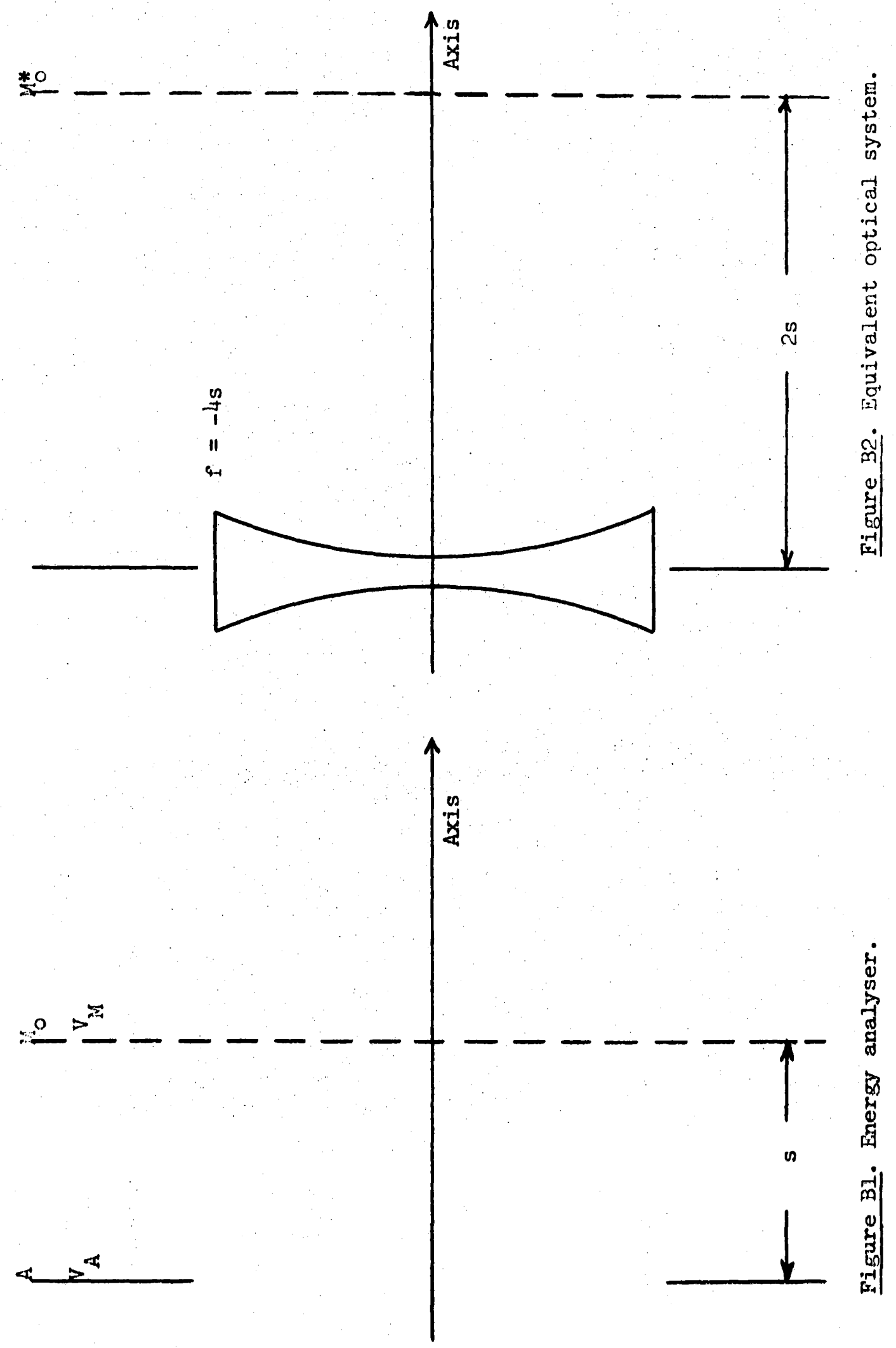
The aperture field acts like a diverging lens of focal length:

$$
f=\frac{4\left(V_{A}-V_{C}\right) s}{-\left(V_{A}-V_{M}\right)}
$$

Since $\mathrm{V}_{\mathrm{M}} \simeq \mathrm{V}_{\mathrm{C}}$

$$
f=-4 s
$$

The uniform field between the electrodes causes the electrons to travel on parabolic paths with vertices at $M_{0}$ approximately. These paths when referred to the potential of the end electrode can be replaced by their tangents just inside the aperture lens. The distance between the end electrode and the mesh as indicated by the tangents is $2 s$, twice the actual distance. Figure BI can therefore be replaced by the optical counterpart, Figure B2, in which the rays inside the analyser are atraight. The nesh $M_{6}$ is the same size as $\mathrm{M}_{0}$. Parabolic paths which have their vertices at a point in $M_{0}$ therefore have initial tangents which focus at $M_{0}^{*}$, a distance $s$ beyond $M_{0}$. Since electrons entering the analyser are affected by the diverging action of the aperture field, a pencil for which the tangents inside the analyser are to be focused at $M_{0}^{*}$, must be directed from outside the analyser toward a focus at $M$ (see Figure B3). The position of $M$ relative to the aperture lens is given by:

or

$$
(1 / \mathrm{AM})+(1 / \mathrm{f})=(1 / \mathrm{AM} \text { ) }
$$

therefore

$$
(1 / \mathrm{AM})+(1 /-4 s)=(1 / 2 s) \text {, }
$$

The magnification of $M$ is given by:

$$
m=A M / A M B O=2 / 3 \text {. }
$$




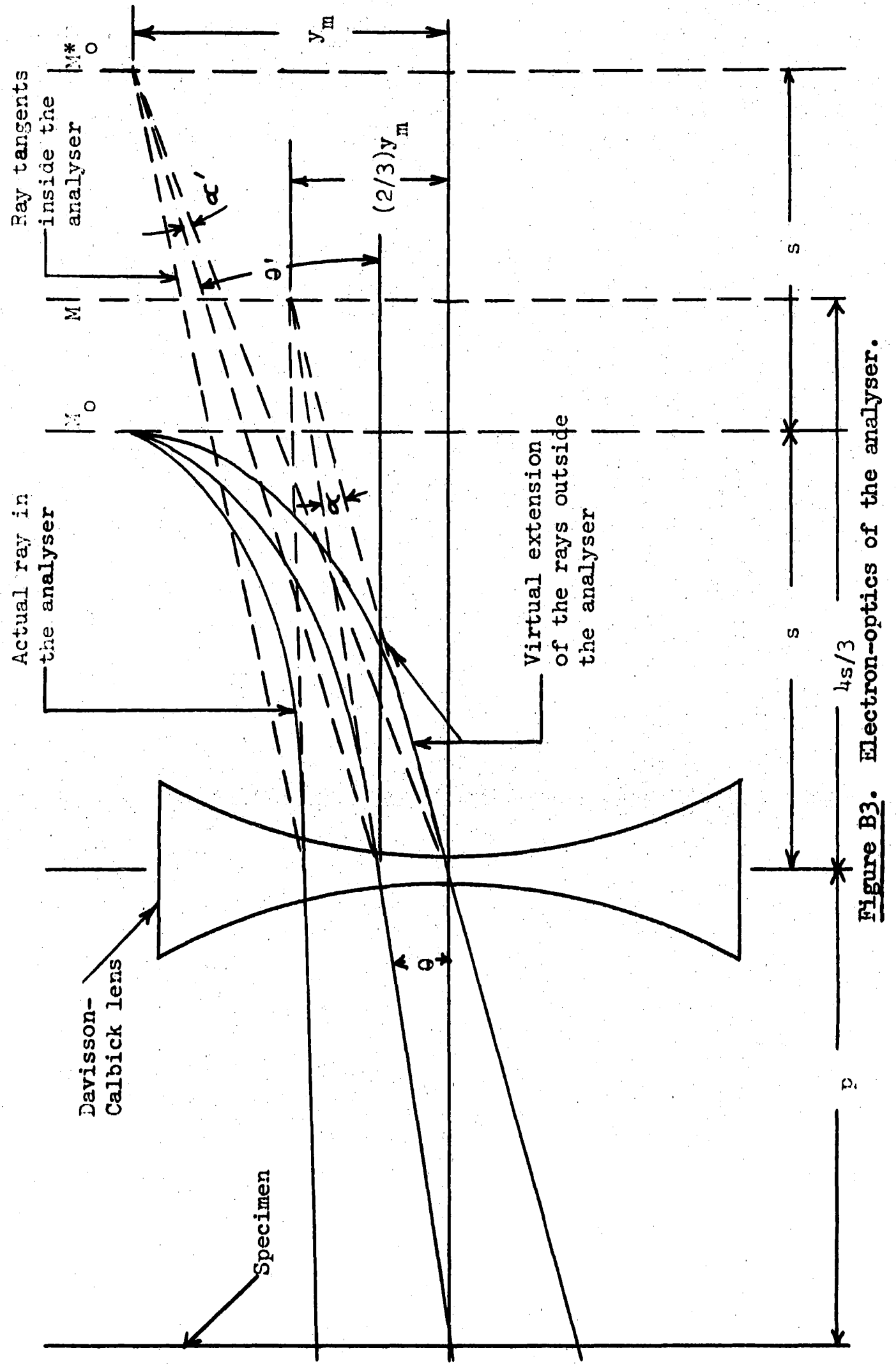


Ihus $\mathrm{H}$, the image of $\mathrm{H}_{0}$ referred to the space outside the analyser, is $2 / 3$ the size of $M_{0}$ and is located within the analyser at a distance $(4 / 3)$ s from the aperture lens.

The angular aperture $\alpha$ of the pencil of rays converging

in the space outside the analyser to a focus at is is also

affected by the action of the aperture lens. The diverging

effect of this lens reduces the angular aperture to

$\alpha^{\prime}=(2 / 3) \propto$ as can be seen from Figure B3. 


\section{APPENDIX C}

\section{FURTHER ASPECTS OF THE ANLIYSER}

The analyser measures the energy associated with the axial velocity of the electrons. The axial beam voltage, defined by ${ }_{3} \mathrm{mv}_{\mathrm{z}}^{2}=\mathrm{eV}_{\mathrm{z}}$, depends on the angle which the electron path makes with the z-axis. In the space outside the analyser the chief ray (the electron path) along the axis of the imaging cone makes an angle $\theta=\rho / p$ with the axis. The aperture lens of the analyser increases the angle to $\theta^{\prime}$, as shown in Figure $\mathrm{Cl}$, given by:

$$
\theta^{\prime}=\theta+p / f \mid
$$

which can be expressed as:

$$
\theta^{\prime}=\theta(1+p / 4 s)
$$

where $\rho=p \theta$ and $f=-4 s$. The beam voltage just inside the analyser is given by:

$$
e V_{B}=e V_{A}-e V_{C}=3 m v^{2}=\frac{1}{z} m v_{z}^{2}+\frac{1}{m} m v_{r}^{2}
$$

Then the loss in axdal beam voltage due to the inclination of the ray is given by:

$$
v_{z}(\text { Ioss })=v_{B}-v_{z}-1 / 2(\mathrm{~m} / \mathrm{e}) v_{r}^{2}
$$

and remains constant as the electron approaches the retarding mesh, since $v_{r}$ is constant inside the analyser. In terms of $\theta^{\prime}$, the axial heam voltage loss for the chief ray is: 

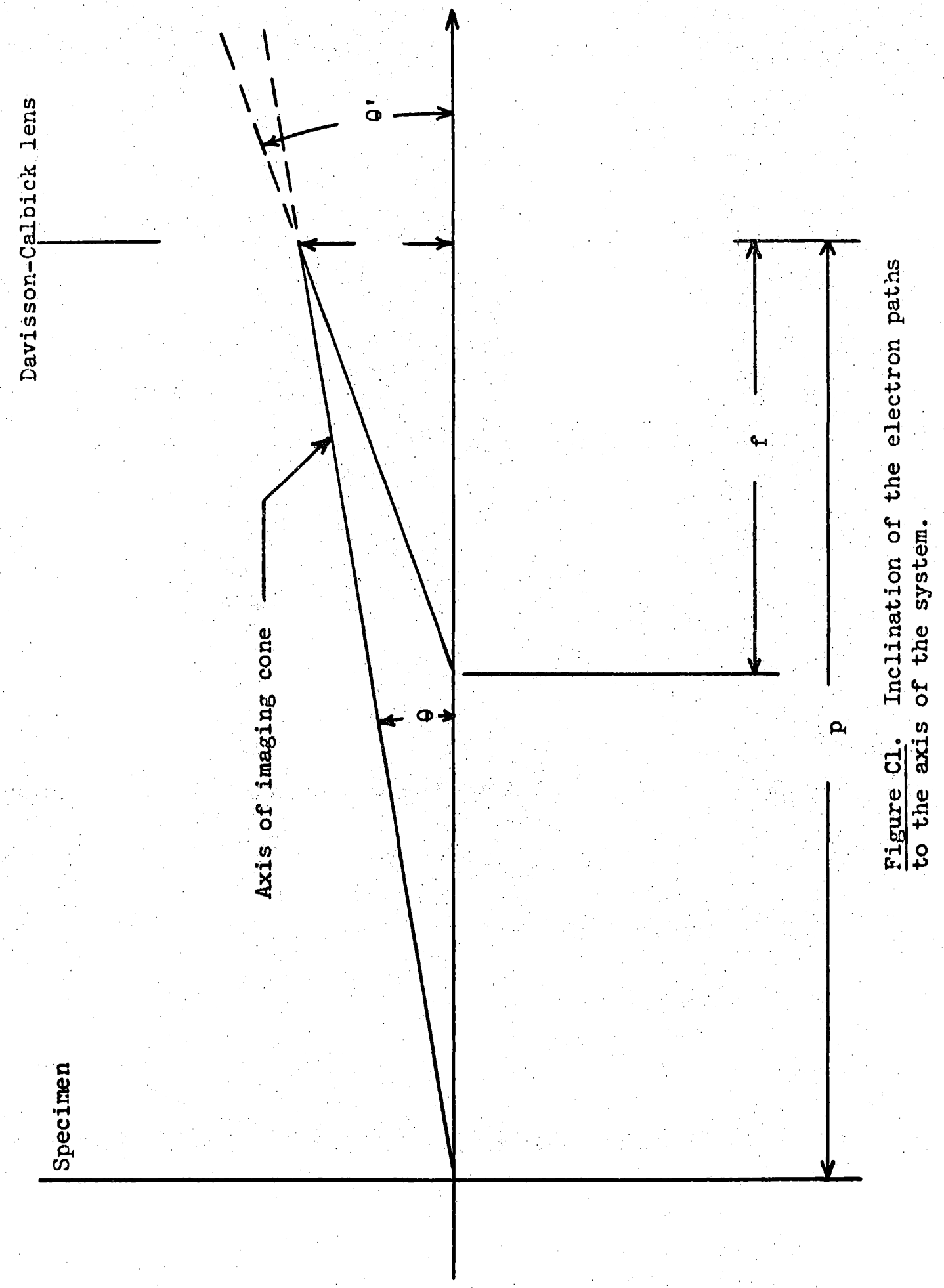

49 


$$
\begin{aligned}
\mathrm{V}_{\mathrm{z}}(\text { loss }) & =1 / 2(\mathrm{~m} / \mathrm{e})(\mathrm{v} \sin \theta)^{2} \\
& =\mathrm{V}_{\mathrm{B}} \sin ^{2} \theta \\
& =\mathrm{V}_{\mathrm{B}} \theta^{\prime}
\end{aligned}
$$

or in terms of $\theta$ :

$$
V_{z}(\text { loss })=V_{B} \theta^{2}(I+p / 4 s)^{2} .
$$

In the pencil of rays converging to $\mathbb{M}_{0}^{*}$ the angle to the z-axis varies in magnitude from $\theta^{\prime}-\alpha^{\prime}$ to $\theta^{\prime}+\alpha^{\prime}$ for $\theta^{\prime}>\alpha^{\prime}$ or from zero to $\theta^{\prime}+\alpha^{\prime}$ for $\theta^{\prime} \leq \alpha^{\prime}$. The corresponding range in axial beam voltage loss is:

for $\theta^{\prime}>\alpha^{\prime}$; and

$$
V_{z}(\text { loss })=V_{B}\left[\left(\theta^{\prime}+\alpha^{\prime}\right)^{2}-\left(\theta^{\prime}-\alpha^{\prime}\right)^{2}\right]=4 V_{B} \theta^{\prime} !
$$

$$
=v_{B}\left(\theta^{\prime}+\alpha^{\prime}\right)^{2}
$$

for $\theta^{\prime} \leqslant \alpha^{\prime}$. 